\title{
Subdomain BEM formulations for the solution of bio-heat problems in biological tissue with melanoma lesions
}

\author{
J. Iljaž ${ }^{* 1}$, L. C. Wrobel ${ }^{2}$, M. Hriberšek ${ }^{1}$ and J. Marn ${ }^{1}$ \\ 1 - Faculty of Mechanical Engineering, University of Maribor \\ Smetanova 17, SI-2000 Maribor, Slovenia, * corresp: jurij.iljaz@um.si \\ 2 - Brunel University London, Kingston Lane, Uxbridge, UB8 3PH, United Kingdom
}

June 25, 2017

\begin{abstract}
The main objective of the research presented in this paper is the development of efficient subdomain BEM solvers for the solution of steady-state and transient bio-heat problems in biological tissue, particularly involving melanoma of different sizes such as Clark II and IV. The short-term goal of the work is to investigate which of the numerical schemes implemented here produces the highest accuracy and efficiency, as a first step towards the long-term goal of solving inverse bio-heat problems for tumour diagnostics, i.e. the detection of tumour size and tumour parameters. The numerical results show that quadratic elements produce high accuracy with coarser meshes, and are thus more computationally efficient for this type of problem. It was also found that, for transient problems, a BEM formulation using the time-dependent fundamental solution of the diffusion equation was more efficient than the use of the fundamental solution of the Laplace equation with a finite difference time discretisation scheme, as much larger time steps could be used for the same accuracy. This work proposes that the subdomain BEM with quadratic elements and a time-dependent fundamental solution provides high accuracy and reduced computational time, and is thus indicated for the inverse analysis of bio-heat problems.
\end{abstract}

Keywords: Bio-heat, melanoma, subdomain Boundary Element Method, timedependent fundamental solution, quadratic elements.

\section{Introduction}

Heat and mass transport phenomena are generally relevant to numerous processes in the field of mechanical engineering, energy, bioengineering, as well as medicine. This work focuses on bio-heat transfer phenomena in biological tissue. The main applications of this work are in tumour detection or blood perfusion reconstruction $[1,2,3,4,5$, 
$6,7]$, cryosurgery $[8,9,10,11]$, hyperthermia $[12,13,14,15]$, laser treatment $[16,17$, 18], thermoregulation simulations $[19,20,21,22]$, etc. We aim to simulate naturally occurring problems of heat transfer in order to evaluate the tissue response. In the past, we have shown $[23,24,25]$ that the results of simulations depend on many factors such as computational discretization, numerical method used, governing equations and model, yet the numerical accuracy largely remains overlooked although it may significantly influence the quality of the results.

This question, of course, is dependent on the nature of the problem under investigation. For example, we do not foresee the numerical accuracy of simulations posing an important obstacle in thermal treatments like cryosurgery or hyperthermia, where the error can be in the range of $5 \%$. However, it is very important in the field of diagnostic like tumour detection, where numerical simulations of heat transfer are compared to measurements of an actual bio-heat problem. These types of problems, commonly referred to as inverse problems, are solved by comparing results of a numerical model to actual experimental measurements to obtain a set of initial parameters on which progress or existence of the condition of the tissue can be ascertained. Of course, there is always a difference between the model and experiment, such as numerical errors, measurement noise and intrinsic model uncertainties. Hence, the problem transforms into one of optimization, more precisely, the estimation of an objective function. Large numerical errors result in erroneous estimated objective functions for a certain set of searched parameters. Low numerical error, on the other hand,results in a smooth and well defined objective function. In most cases, especially in the field of inverse bio-heat problems, the objective function is determined numerically and is known only for discrete values of the searched parameters. This presents yet another obstacle in the solution of inverse problems, especially if a deterministic optimization method is used where the objective function has to be smooth in order to apply correct gradients to the solution.

The experimental work was based on thermal imaging. Nowadays, thermal imaging cameras have a resolution of $640 \times 480$ pixels with sensitivity of $<30 \mathrm{mK}$, which means that the thermal recording or measurement of skin surface temperature can be both fine and precise. The sensitivity of measurements is lower than $0.03 K$, hence providing very high accuracy.

This paper shows results of transient bio-heat problems where tissue is modeled with different layers and melanoma of different sizes. The numerical technique adopted is the Boundary Element Method (BEM), which allows for simultaneous evaluation of temperature and heat flux. It should be noted that, in addition to an accurate solver, we are looking for speed of calculation prompting the use of the subdomain approach which was shown to be much faster than the classical BEM, see Ramšak and Škerget $[24,25]$ and Ravnik et al. [26, 27]. Thus, another aim of this paper is to find an efficient solver satisfying both the accuracy and the computational speed requirements. The BEM or the Dual Reciprocity BEM for solving bio-heat problems has already been used in works of Luna et al. [2], Partridge and Wrobel [4, 6], Multiple Reciprocity BEM has been used by Paruch and Majchrzak [5], limited to steady-state conditions, and in our previous work for solving inverse bio-heat problems [28]. This work is focused on presenting results for transient bio-heat problems comparing different element types: linear and quadratic elements, as well as different formulations: elliptic where the Laplace fundamental solution is coupled with a finite difference (FD) time marching scheme, and a parabolic formulation where the time-dependent fundamental solution to the diffusion equation is 
used. The time-dependent fundamental solution has already been used by Yan et al. [29] for one dimensional inverse heat source problems, as well as by Young et al. [30] and Johansson and Lesnic [31] in a MFS approach for general homogenous parabolic problems. This work develops a fast and accurate numerical scheme by coupling the subdomain BEM with a time-dependent fundamental solution for solving transient bioheat melanoma problems considering heterogeneous tissue. The paper also shows the numerical accuracy of the results for simulating the cooling-rewarming test proposed by Çetingül and Herman [3] and Cheng and Herman [32], where four test examples using Clark II and Clark IV melanoma sizes and different melanoma blood perfusion rates are proposed.

The paper is organized as follows: Section 2 presents the governing equation and the framework for the melanoma test problems, Section 3 describes the subdomain BEM approach and numerical schemes adopted, together with different numerical formulations, Section 4 covers the transient test examples, material properties, computational mesh and boundary conditions. Section 5 discusses steady-state and transient results, numerical accuracy of the elements adopted and proposed formulations, as well as a comparison of computational speed. The paper closes with Section 6 that summarizes this work and emphasizes the importance of its results.

\section{Governing equation}

There are many bio-heat models that describe heat transfer in tissue, from the simplest macro-scale models $[33,34]$ to more complex models $[35,36,37]$ that include convection or non-Fourier heat transfer effects. The most common bio-heat model is the Pennes model due to its simplicity and robustness, as well as good agreement with experimental and other research work.

The Pennes bio-heat model [33] is written as:

$$
\rho c_{p} \frac{\partial T}{\partial t}=\vec{\nabla} \cdot(k \vec{\nabla} T)+\omega_{b} \rho_{b} c_{p, b}\left(T_{a}-T\right)+q_{m},
$$

where $T$ represents tissue temperature, $\rho, k$ and $c_{p}$ are the effective tissue density, thermal conductivity and specific heat, respectively, $\omega_{b}$ is blood perfusion rate, $\rho_{b}$ blood density, $c_{p, b}$ is specific heat of the blood, $T_{a}$ is arterial blood temperature, $t$ time and $q_{m}$ metabolic heat source. The first term on the left side represents the accumulation of energy, whereas the first term on the right side represents diffusion using a Fourier rheological model. The term which includes the blood perfusion rate represents the heat transfer effect due to the blood flow through the capillary network and can act like a heat source or sink, depending on the correlation between the tissue temperature and arterial blood temperature. The assumption here is that the heat transfer between the surrounding tissue and blood flow happens at the capillary level, because of the very large interface. The last term in the Pennes model represents heat generation due to cell metabolism, which is especially high in the muscle tissue, and therefore acts like a heat source. Between the last two terms, blood perfusion plays a major role in heat generation in the tissue. This is especially noticeable for carcinogenic tissue like tumour that has a higher blood perfusion rate, because of the increased oxygen and nutrition demand, and therefore a higher tissue temperature that can also be observed on the skin surface $[4,2,3,7]$. 
The convection effect due to blood flow is captured in the heat source term. Therefore, the Pennes model is written as a parabolic differential equation and can be described as a diffusion model with a non-linear heat source. The blood flow is replaced by a blood perfusion parameter, which represents the net blood flow rate on the capillary network per tissue volume and does not have direction. The blood perfusion rate is usually treated as constant, as will be in this work; however, it is known to be temperature dependent [4]. The arterial blood temperature is often treated as the body core temperature, which is mostly constant in resting condition. Otherwise, a thermoregulation model is needed for the whole body, to determine the time-dependent body core temperature in various conditions $[19,22]$. In this paper, a constant arterial blood temperature will be considered.

This work considers bio-heat problems with skin melanoma together with the surrounding tissue of different layers. The assumption here is that each layer has constant material properties. Therefore, the non-homogeneous tissue will be treated as a number of space-wise homogeneous components with constant material properties, for which the governing equation (1) can be written in the following form:

$$
\frac{1}{a} \frac{\partial T}{\partial t}=\Delta T+\frac{\omega_{b} \rho_{b} c_{p, b}}{k}\left(T_{a}-T\right)+\frac{q_{m}}{k},
$$

where $a=k / \rho c_{p}$ represents the thermal diffusivity of the tissue and $\Delta$ is the Laplace operator. In this case, the computational domain $\Omega(\vec{r})$ has to be divided into several subdomains $\Omega_{s}(\vec{r})$ that are interconnected through the equilibrium and compatibility interface conditions:

$$
T_{i}(\vec{r}, t)=T_{i+1}(\vec{r}, t), \quad k_{i} \cdot \frac{\partial T_{i}(\vec{r}, t)}{\vec{n}_{i}}=k_{i+1} \cdot \frac{\partial T_{i+1}(\vec{r}, t)}{\vec{n}_{i+1}}
$$

where $\vec{r}$ represents the space vector, $\vec{n}$ a normal to the subdomain boundary and indices $i$ and $i+1$ represent adjoining subdomains. Therefore, the bio-heat transfer for the whole computational domain is described with the set of equations (2), which are written for each individual layer of tissue, and the subdomains are connected through the compatibility and equilibrium conditions (3).

\section{Boundary Element Method}

The Boundary Element Method (BEM) is used to solve the direct bio-heat problem. The main reason for choosing this method is its advance treatment of boundary conditions and the availability of fundamental solutions for its implementation. For instance, Neumann boundary conditions are directly incorporated in the formulation without any additional approximation, which increases the accuracy of the numerical solution. The fundamental solution adopted for the governing equation influences the numerical accuracy, because the parts of the differential equation that are not included in the fundamental solution have to be approximated. The level of accuracy of the BEM is therefore related to the chosen fundamental solution and interpolation functions, which will be discussed in this work.

This paper shows a comparison between the use of steady and transient fundamental solutions and also how different interpolation functions affect the numerical solution and computational time. The subdomain BEM approach has already been discussed in detail in $[24,25,27]$, therefore only a brief description of this technique is presented here. 


\subsection{Elliptic formulation}

The governing equation (2) written for each subdomain $\Omega_{s}(\vec{r})$ can be treated as a nonhomogeneous elliptic equation in the form of a Poisson equation, which for the temperature field $T=T(\vec{r})$ is written as:

$$
\Delta T(\vec{r})=b(\vec{r}),
$$

where $b(\vec{r})$ represents the source term or the non-homogeneous part of the equation. Starting with the integral form of Green's second identity, which in the case of the Poisson equation (4) can be written in the following form:

$$
c(\vec{\xi}) T(\vec{\xi})=\int_{\Gamma_{s}} q(\vec{R}) u^{*}(\vec{\xi}, \vec{R}) d \Gamma-\int_{\Gamma_{s}} T(\vec{R}) q^{*}(\vec{\xi}, \vec{R}) d \Gamma+\int_{\Omega_{s}} b(\vec{r}) u^{*}(\vec{\xi}, \vec{r}) d \Omega_{s},
$$

where $\Omega_{s}$ represents the computational domain and $\Gamma_{s}$ its boundary, $\vec{R}$ is the spatial vector of the boundary, $q=\partial T / \partial n$ is the normal derivative of the temperature, $\vec{\xi}$ represents the position of the source point, $c(\vec{\xi})$ the free coefficient that depends on the position of the source point, and $u^{*}$ and $q^{*}=\partial u^{*} / \partial n$ are the fundamental solution and its normal derivative, respectively.

The fundamental solution for the 2D Laplace equation is:

$$
u^{*}(\vec{\xi}, \vec{r})=-\frac{1}{2 \pi} \cdot \ln [\|\vec{d}(\vec{\xi}, \vec{r})\|],
$$

where $\vec{d}(\vec{\xi}, \vec{r})$ represents the distance vector between the source point and an arbitrary space point, and $\|\cdot\|$ the absolute-value norm. Therefore, the normal derivative of the fundamental solution is:

$$
q^{*}(\vec{\xi}, \vec{r})=\frac{\vec{n} \cdot \vec{d}(\vec{\xi}, \vec{r})}{2 \pi\|\vec{d}(\vec{\xi}, \vec{r})\|^{2}} .
$$

As can be seen from the integral equation (5), we have to evaluate the boundary integrals as well as the domain one, which cannot be avoided in the case of a non-homogeneous elliptic equation. In the case of a homogeneous elliptic equation, the domain integral vanishes and only boundary integrals remain. However, it is possible to transform the domain integral to an equivalent boundary integral by introducing radial basis functions usually adopted in the Dual Reciprocity BEM (DRBEM) [4, 38, 2]. In this case, the accuracy of the numerical solution depends on the chosen radial basis function, the governing equation, as well as the number of boundary and internal points $[4,6]$. In this work, we will evaluate the domain integral directly since the cost for this is not high as we have to evaluate the domain and boundary integration only once, as the mesh is fixed in space and time.

The value of the free coefficient $c(\vec{\xi})$ depends on the position of the source point $\vec{\xi}$, and is defined as

$$
\begin{array}{ll}
c(\vec{\xi})=1, & \vec{\xi} \in \Omega_{s}, \\
c(\vec{\xi})=\beta /(2 \pi), & \vec{\xi} \in \Gamma_{s} .
\end{array}
$$

where $\beta$ represents the solid angle at point $\vec{\xi}$.

To solve the integral equation (5), the computational domain $\Omega_{s}$ and the boundary $\Gamma_{s}$ have to be discretized. Four-node linear cells have been used, and consequently twonode linear elements for the boundary. To test the numerical accuracy of the proposed 
methodology, we tested different interpolation functions for the temperature field, its normal derivative and the source term, as described in subsection 3.3, together with the subdomain BEM approach. The global system of equations for the elliptic Poisson equation (4) is written as

$$
\left[H_{e}\right]\{T\}=\left[G_{e}\right]\{q\}-\left[S_{e}\right]\{b\} .
$$

where $\left[H_{e}\right],\left[G_{e}\right]$ and $\left[S_{e}\right]$ are the matrices obtained using the Laplace fundamental solution, $\{T\}$ is the vector of discrete values of the temperature field, $\{q\}$ is the vector of discrete values of the temperature normal derivative and $\{b\}$ is the vector of discrete values of the non-homogeneous part.

We now apply the above procedure to the bio-heat equation (2), where at first we rewrite the equation in the form of a Poisson equation:

$$
\Delta T=\frac{1}{a} \frac{\partial T}{\partial t}-\frac{\omega_{b} \rho_{b} c_{p, b}}{k}\left(T_{a}-T\right)-\frac{q_{m}}{k},
$$

where the non-homogeneous part $b(\vec{r})$ is now:

$$
b=\frac{1}{a} \frac{\partial T}{\partial t}-\frac{\omega_{b} \rho_{b} c_{p, b}}{k}\left(T_{a}-T\right)-\frac{q_{m}}{k} .
$$

The temperature time derivative is estimated with a second-order finite diference (FD) scheme as [28]:

$$
\frac{\partial T}{\partial t} \approx \frac{3 T\left(t_{F}\right)-4 T\left(t_{F-1}\right)+T\left(t_{F-2}\right)}{2 d t}
$$

where $t_{F}, t_{F-1}$ and $t_{F-2}$ represent different time steps and $d t$ is the time difference between two adjacent time steps. For the first time step, the following assumption has been made; $T\left(t_{F-1}\right)=T\left(t_{F-2}\right)$, because we do not know the temperature field before the initial conditions, which reduces the FD time marching scheme to first-order. Including the approximation (12) into equation (10) and using a fully implicitly scheme $\left(T=T\left(t_{F}\right)\right.$, $\left.q=q\left(t_{F}\right)\right)$, equation (9) is rewritten as:

$$
\begin{gathered}
\left(\left[H_{e}\right]+\left\{\frac{3}{2 a d t}+\frac{\omega_{b} \rho_{b} c_{p, b}}{k}\right\}\left[S_{e}\right]\right)\left\{T\left(t_{F}\right)\right\}=\left[G_{e}\right]\left\{q\left(t_{F}\right)\right\}+\left\{\frac{4}{2 a d t}\right\}\left[S_{e}\right]\left\{T\left(t_{F-1}\right)\right\} \\
-\left\{\frac{1}{2 a d t}\right\}\left[S_{e}\right]\left\{T\left(t_{F-2}\right)\right\}+\left[S_{e}\right]\left\{\frac{\omega_{b} \rho_{b} c_{p, b}}{k} T_{a}+\frac{q_{m}}{k}\right\} .
\end{gathered}
$$

As can be seen, the non-linear part of the perfusion term is now included in the system matrix, which give us the advantage of solving the system of equations only once per time step. This numerical scheme has been used for solving the bio-heat problem discussed in section 4, treating the bio-heat equation as a non-homogeneous elliptic equation.

\subsection{Parabolic formulation}

We can also treat equation (2) for each subdomain as parabolic, in the following general form:

$$
\Delta T(\vec{r}, t)+b(\vec{r}, t)=\frac{1}{a} \frac{\partial T}{\partial t},
$$


where $a$ represents a constant thermal diffusivity and $b(\vec{r}, t)$ the heat source. Starting with the integral form of Green's second identity, which in the case of equation (14) can be written after the time integration in the following form [38]:

$$
\begin{gathered}
c(\vec{\xi}) T\left(\vec{\xi}, t_{F}\right)=a \int_{t_{0}}^{t_{F}} \int_{\Gamma_{s}} q(\vec{R}, t) u_{t}^{*}\left(\vec{\xi}, \vec{R}, t_{F}, t\right) d \Gamma d t-a \int_{t_{0}}^{t_{F}} \int_{\Gamma_{s}} T(\vec{R}, t) q_{t}^{*}\left(\vec{\xi}, \vec{R}, t_{F}, t\right) d \Gamma d t \\
+a \int_{t_{0}}^{t_{F}} \int_{\Omega_{s}} b(\vec{r}, t) u_{t}^{*}\left(\vec{\xi}, \vec{r}, t_{F}, t\right) d \Omega_{s} d t+\int_{\Omega_{s}} T\left(\vec{r}, t_{0}\right) u_{t}^{*}\left(\vec{\xi}, \vec{r}, t_{F}, t_{0}\right) d \Omega_{s},
\end{gathered}
$$

where $t_{0}$ is the initial time and $t_{F}$ the final simulation time, $T\left(\vec{r}, t_{0}\right)$ represents the initial temperature field, and $u_{t}^{*}$ and $q_{t}^{*}$ the time-dependent fundamental solution and its normal derivative, respectively. The free coefficient $c(\vec{\xi})$ remains the same as in the elliptic formulation and is defined by equation (8).

The time-dependent fundamental solution for 2D diffusion problem is defined as [38, 31]:

$$
u_{t}^{*}\left(\vec{\xi}, \vec{r}, t_{F}, t\right)=\frac{1}{4 \pi a \tau} \cdot \exp \left(-\frac{\|\vec{d}(\vec{\xi}, \vec{r})\|^{2}}{4 a \tau}\right),
$$

where $\tau=t_{F}-t$ is the time difference between the final and current time, and the normal derivative is $[38,31]$ :

$$
q_{t}^{*}\left(\vec{\xi}, \vec{r}, t_{F}, t\right)=\frac{\vec{n} \cdot \vec{d}(\vec{\xi}, \vec{r})}{8 \pi(a \tau)^{2}} \cdot \exp \left(-\frac{\|\vec{d}(\vec{\xi}, \vec{r})\|^{2}}{4 a \tau}\right) .
$$

In this work, we adopt a formulation that integrates only over the current time step and not the whole time domain. Therefore, $t_{F}$ represents the final time in each individual time step and $t_{0}=t_{F-1}$, thus $t_{F}-t_{0}=d t$. The advantage is that the time integration can be done only once if the time step remains constant during the simulation, otherwise the time integrals have to be evaluated again. In our case, because the computational mesh does not change with time, the time and space integrals have to be evaluated only once.

The temperature field, its normal derivative, as well as the heat source in equation (15) all depend on time, for which we have to introduce time interpolation functions. We use constant time interpolation for the temperature normal derivative $q(\vec{R}, t)=q\left(\vec{R}, t_{F}\right)$ and the source part $b(\vec{r}, t)=b\left(\vec{r}, t_{F}\right)$, while for the temperature field we use a linear interpolation function [38], written as:

$$
T(\vec{r}, t)=\frac{t_{F}-t}{d t} \cdot T\left(\vec{r}, t_{0}\right)+\frac{t-t_{0}}{d t} \cdot T\left(\vec{r}, t_{F}\right) .
$$

The integral equation (15), considering the time interpolation functions and changing the order of integration, can now be rewritten in the following form:

$$
\begin{gathered}
c(\vec{\xi}) T\left(\vec{\xi}, t_{F}\right)=\int_{\Gamma_{s}} q\left(\vec{R}, t_{F}\right) a \int_{t_{0}}^{t_{F}} u_{t}^{*}\left(\vec{\xi}, \vec{R}, t_{F}, t\right) d t d \Gamma \\
-\int_{\Gamma_{s}} T\left(\vec{R}, t_{0}\right) \frac{a}{d t} \int_{t_{0}}^{t_{F}}\left(t_{F}-t\right) \cdot q_{t}^{*}\left(\vec{\xi}, \vec{R}, t_{F}, t\right) d t d \Gamma-\int_{\Gamma_{s}} T\left(\vec{R}, t_{F}\right) \frac{a}{d t} \int_{t_{0}}^{t_{F}}\left(t-t_{0}\right) \cdot q_{t}^{*}\left(\vec{\xi}, \vec{R}, t_{F}, t\right) d t d \Gamma
\end{gathered}
$$




$$
+\int_{\Omega_{s}} b\left(\vec{r}, t_{F}\right) a \int_{t_{0}}^{t_{F}} u_{t}^{*}\left(\vec{\xi}, \vec{r}, t_{F}, t\right) d t d \Omega_{s}+\int_{\Omega_{s}} T\left(\vec{r}, t_{0}\right) u_{t}^{*}\left(\vec{\xi}, \vec{r}, t_{F}, t_{0}\right) d \Omega_{s}
$$

The time integration of each term inside the boundary and domain integrals can be evaluated analytically [38]:

$$
\begin{gathered}
U_{t}^{*}(\vec{\xi}, \vec{R})=a \int_{t_{0}}^{t_{F}} u_{t}^{*}\left(\vec{\xi}, \vec{R}, t_{F}, t\right) d t=\frac{1}{4 \pi} E_{1}\left(a_{0}\right), \\
Q_{t 1}^{*}(\vec{\xi}, \vec{R})=\frac{a}{d t} \int_{t_{0}}^{t_{F}}\left(t_{F}-t\right) \cdot q_{t}^{*}\left(\vec{\xi}, \vec{R}, t_{F}, t\right) d t=\frac{\vec{n} \cdot \vec{d}(\vec{\xi}, \vec{r})}{8 \pi a d t} E_{1}\left(a_{0}\right), \\
Q_{t 2}^{*}(\vec{\xi}, \vec{R})=\frac{a}{d t} \int_{t_{0}}^{t_{F}}\left(t-t_{0}\right) \cdot q_{t}^{*}\left(\vec{\xi}, \vec{R}, t_{F}, t\right) d t=\frac{\vec{n} \cdot \vec{d}(\vec{\xi}, \vec{r})}{8 \pi a d t}\left[\frac{1}{a_{0}} \exp \left(-a_{0}\right)-E_{1}\left(a_{0}\right)\right],
\end{gathered}
$$

where $E_{1}$ represents the exponential integral function and $a_{0}$ is a parameter defined as:

$$
a_{0}=a_{0}(\vec{\xi}, \vec{r})=\frac{\|\vec{d}(\vec{\xi}, \vec{r})\|^{2}}{4 a d t} .
$$

The initial value of the fundamental solution $u_{t}^{*}\left(\vec{\xi}, \vec{r}, t_{F}, t_{0}\right)$ can be written in a similar manner as in equation (16):

$$
U_{t 0}^{*}(\vec{\xi}, \vec{r})=u_{t}^{*}\left(\vec{\xi}, \vec{r}, t_{F}, t_{0}\right)=\frac{1}{4 \pi a d t} \cdot \exp \left(-a_{0}\right) .
$$

To evaluate the domain and boundary integrals in equation (19) analytically, we use the following approximation of the exponential integral function $E_{1}$ [39]:

$$
E_{1}\left(a_{0}\right) \approx \frac{\exp \left(-a_{0}\right) \cdot \ln \left[1+\frac{G}{a_{0}}+\frac{1-G}{\left(v+z a_{0}\right)^{2}}\right]}{G+(1-G) \cdot \exp \left(\frac{-a_{0}}{1-G}\right)}
$$

where $G$ is a parameter defined as: $G=\exp (-\gamma), \gamma=0.5772156649015328606$ is the Euler constant, $z$ is a constant defined as $z=\sqrt{2(1-G) /\left(2 G-G^{2}\right)}$ and $v=v\left(a_{0}\right)$ is a function defined as:

$$
v\left(a_{0}\right)=\frac{1}{1+a_{0} \sqrt{a_{0}}}+\frac{v_{\infty} m}{1+m}
$$

where $m=m\left(a_{0}\right)$ is

$$
m\left(a_{0}\right)=\frac{20}{47} a_{0}^{\sqrt{31 / 26}}
$$

and $v_{\infty}$ is

$$
v_{\infty}=\frac{(1-G)\left(G^{2}-6 G+12\right)}{3 G(2-G)^{2} z} .
$$

Including the expressions (20)-(22) and (24) into the integral equation (19) we obtain:

$$
\begin{gathered}
c(\vec{\xi}) T\left(\vec{\xi}, t_{F}\right)=\int_{\Gamma_{s}} q\left(\vec{R}, t_{F}\right) U_{t}^{*}(\vec{\xi}, \vec{R}) d \Gamma-\int_{\Gamma_{s}} T\left(\vec{R}, t_{0}\right) Q_{t 1}^{*}(\vec{\xi}, \vec{R}) d \Gamma \\
-\int_{\Gamma_{s}} T\left(\vec{R}, t_{F}\right) Q_{t 2}^{*}(\vec{\xi}, \vec{R}) d \Gamma+\int_{\Omega_{s}} b\left(\vec{r}, t_{F}\right) U_{t}^{*}(\vec{\xi}, \vec{r}) d \Omega_{s}+\int_{\Omega_{s}} T\left(\vec{r}, t_{0}\right) U_{t 0}^{*}(\vec{\xi}, \vec{r}) d \Omega_{s}
\end{gathered}
$$


Integral equation (29) is very similar to equation (5), where only boundary and domain integration is needed as in the elliptic formulation. The domain and boundary discretization schemes remained the same as for the elliptic formulation, as well as the subdomain BEM approach, producing the following global system of equations:

$$
\left[H_{p 2}\right]\left\{T\left(t_{F}\right)\right\}+\left[H_{p 1}\right]\left\{T\left(t_{0}\right)\right\}=\left[G_{p}\right]\left\{q\left(t_{F}\right)\right\}+\left[S_{p}\right]\left\{b\left(t_{F}\right)\right\}+\left[S_{p 0}\right]\left\{T\left(t_{0}\right)\right\},
$$

where $\left[H_{p 2}\right],\left[H_{p 1}\right],\left[G_{p}\right],\left[S_{p}\right]$ and $\left[S_{p 0}\right]$ are matrices obtained by using the time-dependent fundamental solution, $\left\{T\left(t_{F}\right)\right\}$ and $\left\{T\left(t_{0}\right)\right\}$ are vectors of discrete values of the temperature field at the end and at the beginning of the time step, respectively, $\left\{q\left(t_{F}\right)\right\}$ is the vector of discrete values of the temperature normal derivative at the end of the time step and $\left\{b\left(t_{F}\right)\right\}$ is the vector of discrete values of the heat source at the end of the time step.

We can now apply the numerical scheme (30) to the bio-heat equation (2), where $b(\vec{r}, t)$ is defined as:

$$
b(\vec{r}, t)=\frac{\omega_{b} \rho_{b} c_{p, b}}{k}\left(T_{a}-T(\vec{r}, t)\right)+\frac{q_{m}}{k} .
$$

Implementing the source term in equation (31) into the system of equations (30) and substituting the initial time $t_{0}$ of each individual time step by $t_{F-1}$, we obtain the system of equations that solves bio-heat problems using the time-dependent fundamental solution:

$$
\begin{gathered}
\left(\left[H_{p 2}\right]+\frac{\omega_{b} \rho_{b} c_{p, b}}{k}\left[S_{p}\right]\right)\left\{T\left(t_{F}\right)\right\}=\left[G_{p}\right]\left\{q\left(t_{F}\right)\right\} \\
+\left(\left[S_{p 0}\right]-\left[H_{p 1}\right]\right)\left\{T\left(t_{F-1}\right)\right\}+\left[S_{p}\right]\left\{\frac{\omega_{b} \rho_{b} c_{p, b}}{k} T_{a}+\frac{q_{m}}{k}\right\} .
\end{gathered}
$$

As can be seen, part of the temperature-dependent heat source is included in the system matrix, similarly to the elliptic formulation, therefore the system of equations only needs one iteration for each time step. The matrices are evaluated only in the first time step and do not change during the simulation, as the time step is kept constant, otherwise the matrices should be calculated again, which will increase the computational time. The number of operations is similar to the elliptic formulation.

The numerical scheme (32) generated the best results for the bio-heat problem. However, we also tested different time interpolation schemes for the temperature normal derivative, as well as for the temperature-dependent source term, which did not produce the same accuracy and are therefore omitted from this paper.

\subsection{Subdomain BEM approach}

A detailed description of the subdomain BEM approach is given in the work of Ramšak and Škerget [24, 25]; here, only a brief description of the method will be given. The reason for using this approach is that it provides a much faster computational time than the classical BEM where the matrices are fully populated. The system matrix in the subdomain BEM approach becomes sparse and diagonally dominant. The idea behind this approach is treating each cell of the computational mesh as one subdomain and writing the discrete system of equations for every cell individually. Therefore, the source point $\vec{\xi}$ is placed only on the nodes of the cell and the discrete form of the governing equation is written just for the observed cell; the boundary and domain in this case represent the boundary and the area of the cell, respectively. The systems of equations for every cell of the computational mesh are then connected together through compatibility and 
equilibrium conditions on the boundary between two adjoining cells. The main difference between the classical BEM and the subdomain BEM approach is in the treatment of the boundary and domain, where in the classical approach the source point has information about the whole computational domain, while in the subdomain approach it has only information about the individual cell.

To evaluate the boundary and domain integrals in equations (5) and (29) the interpolation functions have been introduced for the boundary approximation of temperature and its normal derivative, as well as for the domain approximation of the source term. To evaluate which numerical scheme works best for the tested problems, we used two different sets of interpolation functions. The first one uses linear continuous interpolation for the boundary temperature $T(\vec{R})$ and domain source function $b(\vec{r})$, and constant interpolation for the temperature normal derivative at the boundary $q(\vec{R})$, representing a linear element. The second set of interpolation functions has the same interpolation for $q(\vec{R})$, but quadratic continuous interpolation for $T(\vec{R})$ and $b(\vec{r})$, representing a quadratic element. The linear and quadratic 2D elements are shown in Figure 1, together with the discrete points for the source, temperature and its normal derivative.

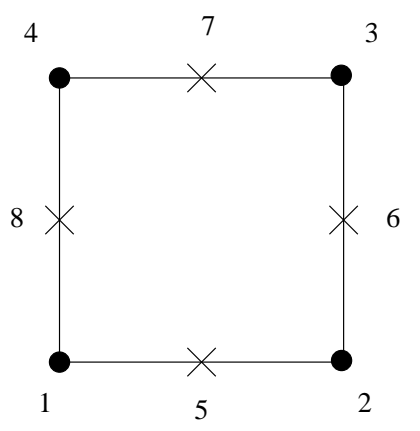

a)

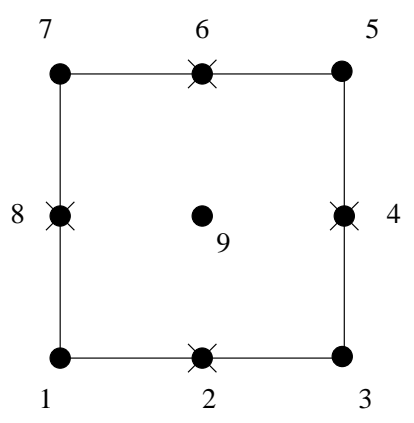

b)

Figure 1: Element types: a) linear and b) quadratic element. Black points indicate the locations of discrete values of $T(\vec{r})$ and $b(\vec{r})$, while crosses indicate the locations of discrete values of $q(\vec{r})$.

The constant interpolation function for the approximation of the normal derivative of the temperature has been chosen to avoid the corner problem and has been used for both elements.

Therefore, the matrix coefficients of system (9) are:

$$
h_{e, i, j}^{n}=\int_{\Gamma_{j}} \phi_{n} q^{*} d \Gamma_{j}, \quad g_{e, i, j}=\int_{\Gamma_{j}} u^{*} d \Gamma_{j}, \quad s_{e, i, j}^{n}=\int_{\Omega_{j}} \phi_{n} u^{*} d \Omega_{j}
$$

where $\phi_{n}$ represent the interpolation functions and indices $i$ and $j$ the position of the source point and element or cell number, respectively. Similarly, the matrix coefficients of system (30) can be written as:

$$
\begin{gathered}
h_{p 1, i, j}^{n}=\int_{\Gamma_{j}} \phi_{n} Q_{t 1}^{*} d \Gamma_{j}, \quad h_{p 2, i, j}^{n}=\int_{\Gamma_{j}} \phi_{n} Q_{t 2}^{*} d \Gamma_{j}, \quad g_{p, i, j}=\int_{\Gamma_{j}} U_{t}^{*} d \Gamma_{j}, \\
s_{p, i, j}^{n}=\int_{\Omega_{j}} \phi_{n} U_{t}^{*} d \Omega_{j}, \quad s_{p 0, i, j}^{n}=\int_{\Omega_{j}} \phi_{n} U_{t 0}^{*} d \Omega_{j} .
\end{gathered}
$$


Applying the interpolation functions and evaluating the integrals, the algebraic or discrete form of the integral equation is obtained. In order to obtain the local system of equations for a given cell or element, the discrete equation is written by positioning the source point in every discrete point for the temperature field. For the linear element, these points are 1-4 and for the quadratic element 1-9. Applying this rule to every cell, the system of equations for the computational domain $\Omega$ or subdomain $\Omega_{s}$ is obtained by using the compatibility and equilibrium conditions for adjoining cells. In the final step, the systems of equations obtained for every subdomain $\Omega_{s}$ are combined in the global system of equations (9) or (30) through the interface conditions between adjoining subdomains.

\section{Computational examples}

The aim of this work is to investigate how different numerical schemes affect the numerical accuracy of solution of direct bio-heat problems under steady-state and transient conditions. We aim to reduce the numerical error to a minimum when solving inverse bio-heat problems by developing robust, accurate and fast numerical schemes suitable for this task.

The computational examples of melanoma problems are based on the works of Luna et al. [2], Bhowmik and Repaka [1] and Çetingül and Herman [3] to provide for a solid comparison basis. Unfortunately, all authors treated the melanoma problem a little differently, by using different boundary conditions, computational domain, as well as material properties and size of the melanoma, therefore a direct comparison is not always feasible. We tested the proposed numerical approach on 2D melanoma problems, including different tissue layers, different melanoma size and different physiological melanoma properties under steady-state conditions and a transient cooling-rewarming test introduced by Çetingül and Herman [3].

The representative computational domain is shown in Figure 2, while global dimensions and thicknesses (dimension in $y$ direction) of different tissue layers are gathered in Table 1. The numerical accuracy has been tested on two different sizes of melanoma, Clark II and Clark IV, which were placed in the middle of the horizontal direction of the computational domain. The size of melanoma is gathered in Table 1, where Clark II melanoma lies in the papillary dermis while Clark IV is already protruding into the fat layer, as can be seen from Figure 2 .

Table 1 also contains the material properties, which are taken from Çetingül and Herman [3] and Luna et al. [2]. These authors also discuss the accuracy of data for melanoma, especially for blood perfusion rate and metabolic heat generation, which are not available in the literature and cannot be measured directly. For these reasons, they also made different simulations by changing the melanoma blood perfusion rate and metabolic heat. They noticed that metabolic heat generation does not have such a strong influence on the change of skin surface temperature as blood perfusion rate. Therefore, in this paper, we also investigate the numerical accuracy of the proposed numerical schemes with different blood perfusion rate for melanoma; $\omega_{b, m 1}=0.0063 s^{-1}$ and $\omega_{b, m 2}=0.0315 s^{-1}$ [3].

This work not only investigates subdomain BEM numerical schemes but also proposes benchmark tests in this field. There are in total four test cases with different sizes of melanoma and different melanoma blood perfusion rates. It also covers the skin 


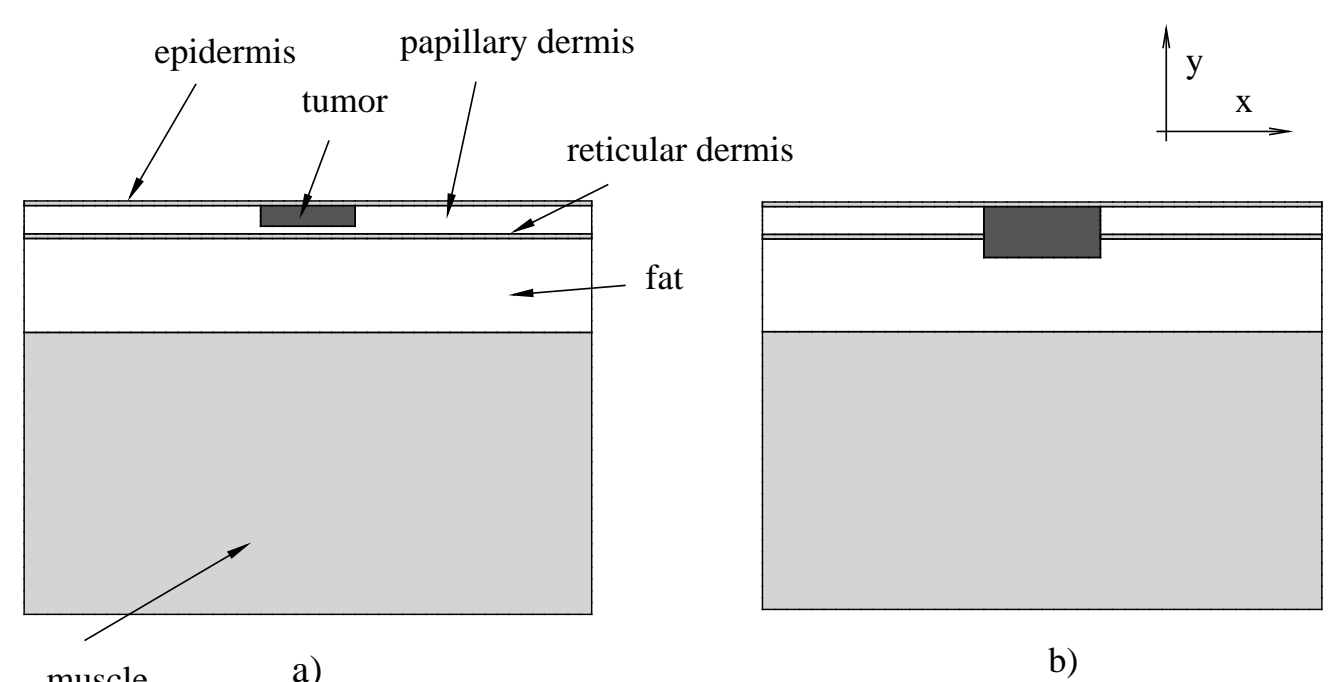

Figure 2: Computational domain: a) Clark II and b) Clark IV size of melanoma.

\begin{tabular}{c|cc|ccccc} 
material & $x[\mathrm{~mm}]$ & $y[\mathrm{~mm}]$ & $\rho\left[\mathrm{kg} / \mathrm{m}^{3}\right]$ & $c_{p}[\mathrm{~J} / \mathrm{kg} k$ & $k[\mathrm{~W} / \mathrm{mK}]$ & $\omega_{b}[1 / \mathrm{s}]$ & $q_{m}\left[\mathrm{~W} / \mathrm{m}^{3}\right]$ \\
\hline epidermis & 12 & 0.1 & 1200 & 3589 & 0.235 & 0.0 & 0.0 \\
papillary dermis & 12 & 0.6 & 1200 & 3300 & 0.445 & 0.0002 & 368.1 \\
reticular dermis & 12 & 0.1 & 1200 & 3300 & 0.445 & 0.0013 & 368.1 \\
fat & 12 & 1.2 & 1000 & 2674 & 0.185 & 0.0001 & 368.3 \\
muscle & 12 & 6.0 & 1085 & 3800 & 0.510 & 0.0027 & 684.2 \\
\hline tumour - Cl. II & 2.0 & 0.44 & 1030 & 3852 & 0.558 & $0.0063 ; 0.0315$ & 3680 \\
tumour - Cl. IV & 2.5 & 1.1 & 1030 & 3852 & 0.558 & $0.0063 ; 0.0315$ & 3680 \\
\hline blood & - & - & 1060 & 3770 & - & - & -
\end{tabular}

Table 1: Material properties with tissue dimensions [2],[3].

temperature steady-state condition, as well as transient skin temperature response to a cooling-rewarming test described by Çetingül and Herman [3].

For a steady-state simulation, we prescribe the Dirichlet boundary condition on the bottom assuming the body core temperature $T=T_{a}=37^{\circ} \mathrm{C}$, while on the sides the Neumann or adiabatic boundary condition $q=0 \mathrm{~W} / \mathrm{m}^{2}$ is prescribed. On the skin surface the Robin boundary condition has been chosen, where the heat transfer coefficient takes into account natural convection, evaporation and radiation; $\alpha=10 \mathrm{~W} / \mathrm{m}^{2} \mathrm{~K}, T_{\infty}=22.4^{\circ} \mathrm{C}$. We obtain the steady-state results using the proposed transient schemes by applying a large time step; $d t=10^{30} \mathrm{~s}$, and zero value initial conditions; $T_{0}=0^{\circ} \mathrm{C}$.

The arterial blood temperature has been taken equal to the mean body core temperature, which is in the range of $36.5^{\circ} \mathrm{C}-37.5^{\circ} \mathrm{C}$ and mostly constant under resting conditions. Therefore, the arterial blood temperature is assumed to be constant during the steady-state and cooling-rewarming tests.

To simulate the cooling-rewarming test, the boundary conditions are the same as for the steady-state simulation, with the exception of the prescribed boundary condition on the skin surface. For the first $60 \mathrm{~s}$ we cool the skin by applying the constant surface 
temperature of $T=13^{\circ} \mathrm{C}$, and after that the skin is exposed to the surrounding environment, which means prescribing the Robin boundary condition of $\alpha=10 \mathrm{~W} / \mathrm{m}^{2} \mathrm{~K}$ and $T_{\infty}=22.4^{\circ} \mathrm{C}$. We simulate the rewarming time of $10 \mathrm{~min}=600 \mathrm{~s}$. For the initial conditions of the cooling-rewarming test, the solution of the steady-state simulation has been used.

A structured mesh generator has been developed to discretize the computational domain shown in Figure 2, which builds up the computational mesh according to the characteristic element size in both directions; $d x$ and $d y$. Figure 3 shows a representative computational mesh, together with a detailed view of the melanoma region. As can be seen, the very thin layers of epidermis and reticular dermis are described with a minimum of two elements, regardless of the element size $d y$, otherwise the temperature distribution would be described as linear when using linear elements.
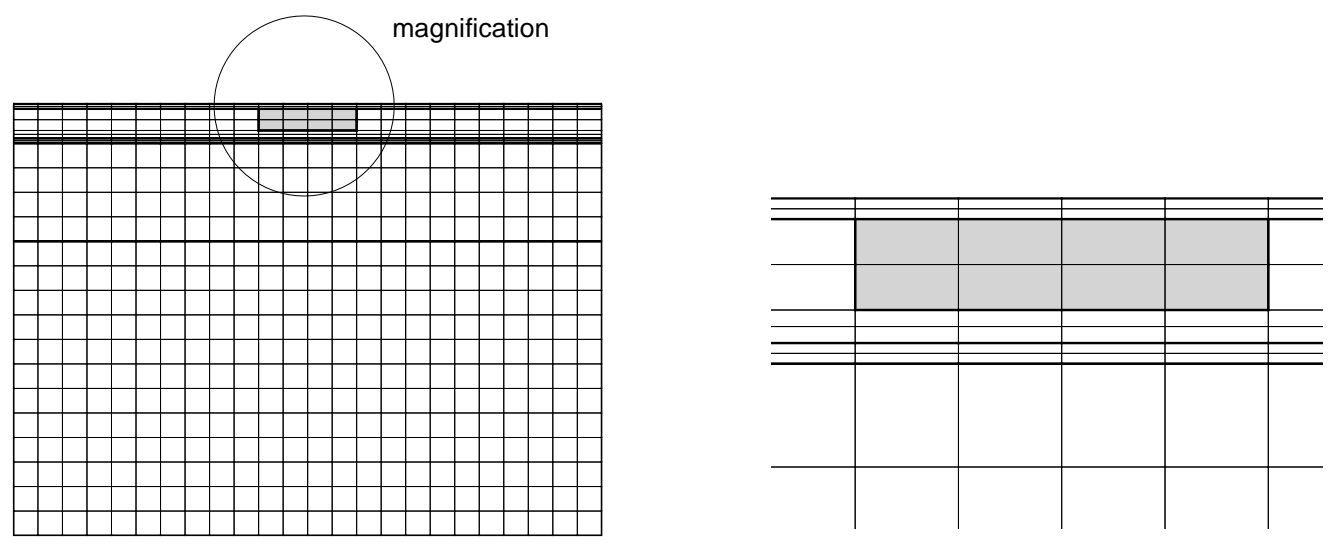

Figure 3: Representative computational mesh of Clark II melanoma using an element size of $x=0.5 \mathrm{~mm}$.

\section{Results and discussion}

Herein, the results of previously discussed problems are given and their discussion is presented. We decided to focus on skin temperature distribution for both the steadystate and transient simulations, as temperature is the actual parameter resulting from diagnostic efforts $[1,2,3]$. Therefore, the results will be presented as graphs for the skin surface temperature, analyzing the numerical accuracy and computational speed for a given test case based on numerical scheme, computational mesh and time step.

The complexity of problems prevent comparison with analytical results. We believe, however, that if a numerical solution is found to be invariant on further refining domain and time discretization this could be used as a basis for comparison, and we call that "near analytical results". The analysis of numerical schemes is divided into two sections, steady-state and transient simulations. In steady-state simulations we test the effect of the chosen element type and element size, while for the transient conditions we test time 
step and the difference between the elliptic and parabolic formulations with quadratic elements.

The CPU time of each simulation is also recorded to investigate the speed-up by using different formulations or different domains and time discretizations. All calculations have been made on a single processor $i 7-920 @ 2.76 \mathrm{GHz}$, therefore the results are comparable.

\subsection{Steady-state results}

The elliptic and parabolic formulations gave the same steady-state results, therefore the results presented in this section are obtained using the elliptic formulation. The main focus of this analysis is to determine the appropriate computational mesh to get meshindependent solutions, which can then be used for the transient simulations.

Figure 4 shows the skin temperature results using linear elements for all four test cases, while using different element sizes where $d x=d y$. There is a distinct difference in the results for elements larger than $d x=0.2 \mathrm{~mm}$, otherwise the difference is not visible and is omitted in the figure. Smaller element sizes have also been used, as can be seen in Table 2, where mesh statistics are presented for Clark II and Clark IV melanoma using linear and quadratic elements.

\begin{tabular}{|c|c|c|c|c|c|c|}
\hline \multirow{2}{*}{$\begin{array}{c}d x \\
{[m m]}\end{array}$} & \multicolumn{3}{|c|}{ Clark II } & \multicolumn{3}{|c|}{ Clark IV } \\
\hline & $\begin{array}{c}\text { number of } \\
\text { elements }\end{array}$ & $\begin{array}{c}\text { linear } \\
\text { number of } \\
\text { nodes }\end{array}$ & $\begin{array}{c}\text { quadratic } \\
\text { number of } \\
\text { nodes }\end{array}$ & $\begin{array}{c}\text { number of } \\
\text { elements }\end{array}$ & $\begin{array}{c}\text { linear } \\
\text { number of } \\
\text { nodes }\end{array}$ & $\begin{array}{c}\text { quadratic } \\
\text { number of } \\
\text { nodes }\end{array}$ \\
\hline 1.0 & 192 & 271 & 938 & 170 & 257 & 846 \\
\hline 0.5 & 576 & 734 & 2,614 & 552 & 708 & 2,512 \\
\hline 0.2 & 2,880 & 3,248 & 12,250 & 2,726 & 3,073 & 11,590 \\
\hline 0.1 & 10,800 & 11,524 & 44,642 & 10,472 & 11,158 & 43,252 \\
\hline 0.05 & 42,000 & 43,437 & 170,868 & 41,760 & 43,132 & 169,776 \\
\hline 0.03 & 115,818 & 118,172 & 468,028 & 116,508 & 118,792 & 470,592 \\
\hline
\end{tabular}

Table 2: Computational mesh properties using linear and quadratic elements for Clark II and Clark IV test cases.

Results using quadratic elements are presented in Figure 5, where we can observe that even the element size of $d x=1.0 \mathrm{~mm}$ produces good results. Comparing results with Figure 4, we can conclude that results using quadratic elements are much more mesh independent. The reason is in the parabolic behaviour of the solution, which is described more accurately by using quadratic interpolation functions.

Figure 6 compares linear and quadratic elements for all four test cases using a small element size; $d x=0.05 \mathrm{~mm}$, which produced mesh-independent results. As can be seen, there is a noticeable difference between linear and quadratic elements, especially for smaller melanoma size or lower blood perfusion rate.

As could be seen from the results, the temperature difference between the tumor and healthy tissue is in the range of $0.03^{\circ} \mathrm{C}-0.2^{\circ} \mathrm{C}$ which is a small variation. Therefore, the temperature gradients are very small in the proximity of the boundary, confirming that the Neumann boundary conditions does not affect the results or that the size of computational domain in $x$-direction is appropriate for the problem. 

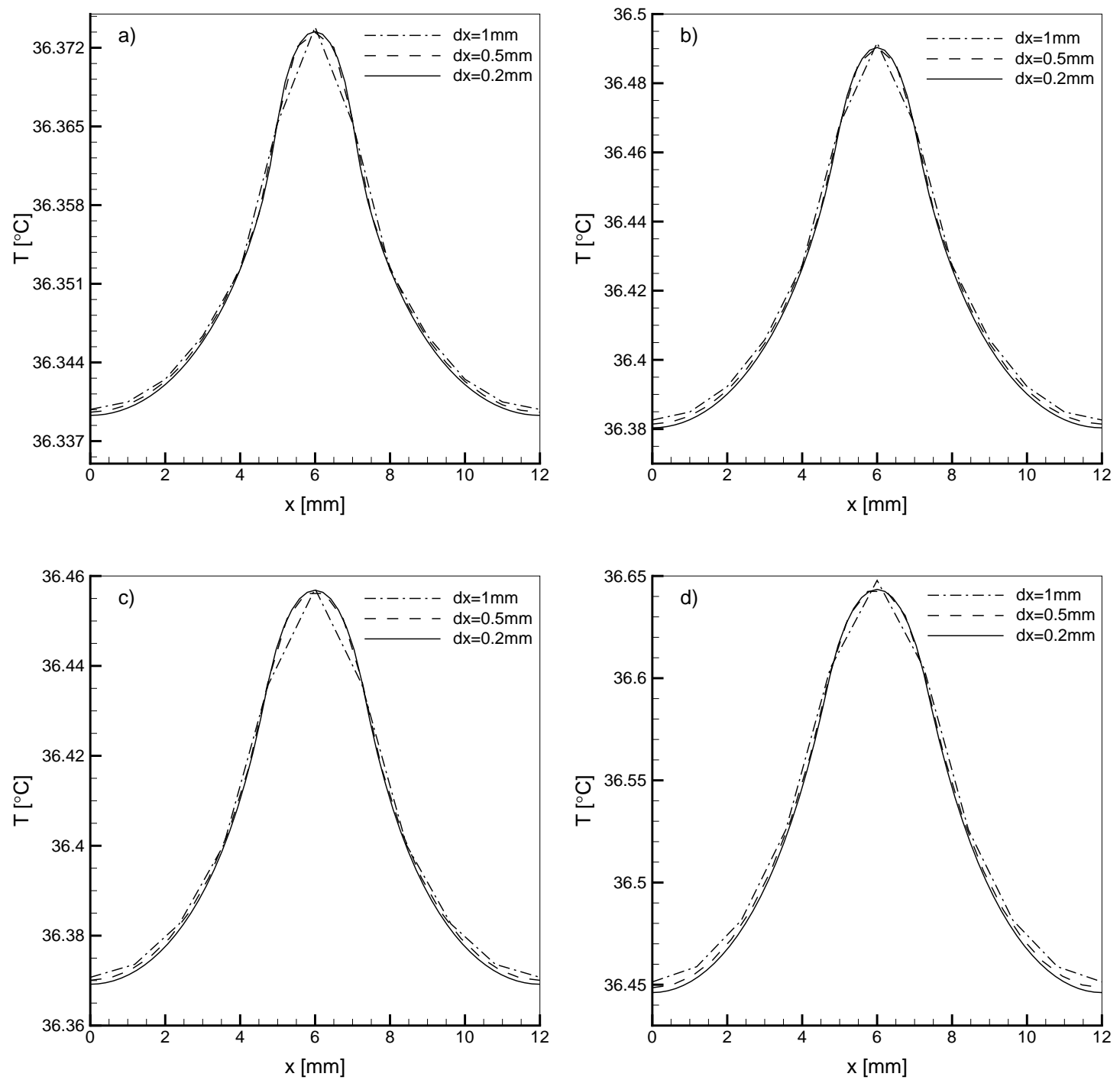

Figure 4: Skin surface temperature using linear elements of different sizes: $1 \mathrm{~mm}, 0.5 \mathrm{~mm}$ and $0.2 m m$, for all test cases; a) Clark II $-\omega_{b, m 1}$, b) Clark II $-\omega_{b, m 2}$, c) Clark IV $-\omega_{b, m 1}$ and d) Clark IV $-\omega_{b, m 2}$.

To better represent the results or to see the numerical difference between element types and mesh sizes, we calculated the $R M S$ error as:

$$
R M S=\sqrt{\frac{1}{n_{j}} \cdot \sum_{j=1}^{n_{j}} \frac{\left(T_{j}-T_{a, j}\right)^{2}}{T_{a, j}^{2}}},
$$

where $n_{j}$ represents the number of discrete values of temperature, $T_{j}$ is the numerically obtained temperature at point $j$ and $T_{a, j}$ the near analytical solution. Table 3 shows the $R M S$ error based on the results obtained using quadratic elements of size $d x=$ $0.03 \mathrm{~mm}$ (best numerical solution). The $R M S$ error for using linear elements is in the range of $10^{-5}$ and reduces when using smaller element sizes; $<d x=0.1 \mathrm{~mm}$, while for quadratic elements the error is substantially lower; $10^{-7}-10^{-6}$, which shows the superior performance of quadratic elements. As can be seen from Table 3 the $R M S$ error for linear elements is not convergent, while quadratic elements show uniform convergence 

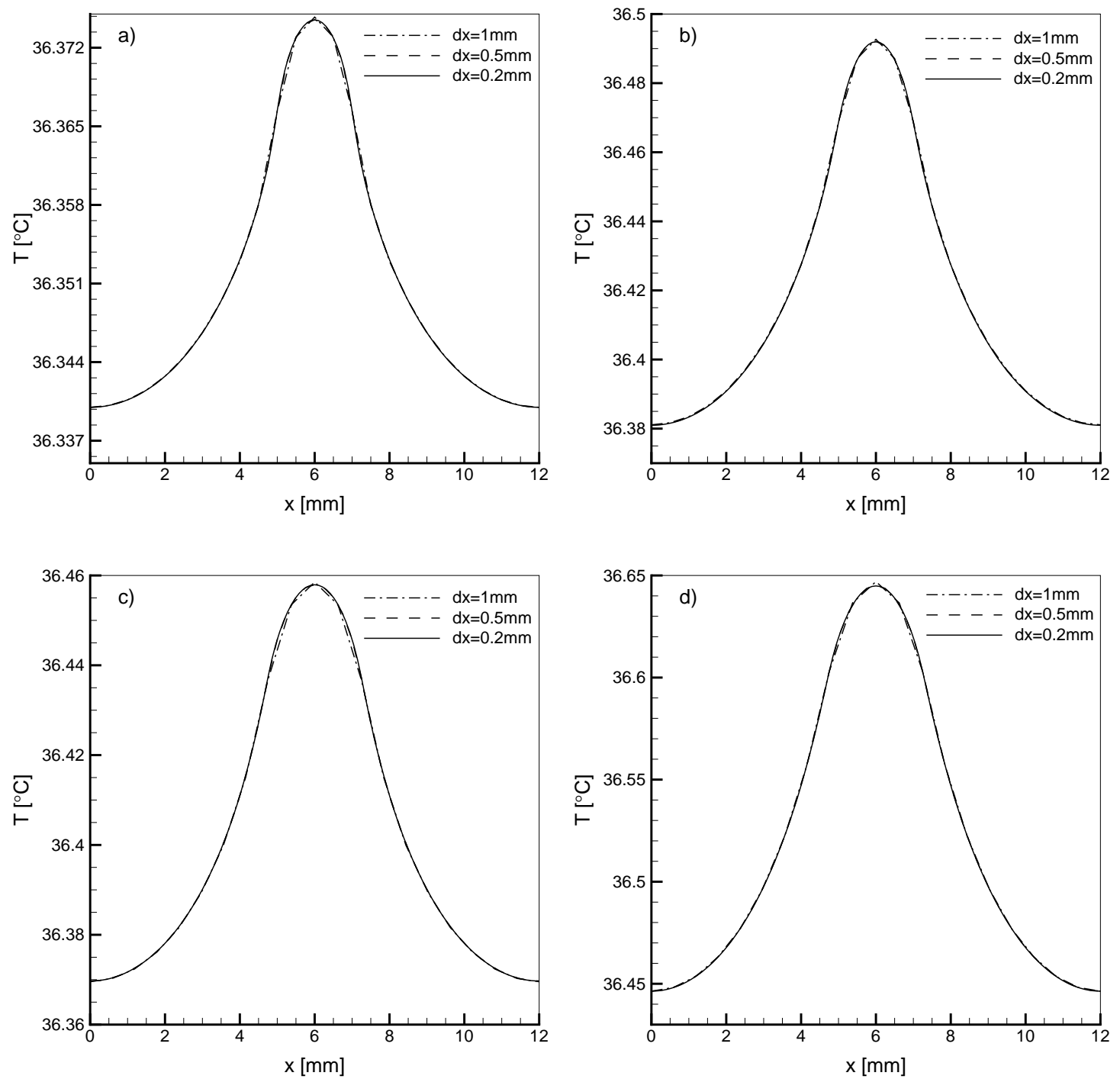

Figure 5: Skin surface temperature using quadratic elements of different sizes: $1 \mathrm{~mm}$, $0.5 \mathrm{~mm}$ and $0.2 \mathrm{~mm}$, for all test cases; a) Clark II $-\omega_{b, m 1}$, b) Clark II $-\omega_{b, m 2}$, c) Clark IV $-\omega_{b, m 1}$ and d) Clark IV $-\omega_{b, m 2}$.

apart from a small and almost negligible difference from the Clark II tumor. It can also be seen from Figure 6 that the linear element solution underestimates the quadratic solution in all the cases.

Table 4 compares the computational time using linear and quadratic elements. The computational time for quadratic elements is longer due to the larger number of BEM nodes. However, a good numerical accuracy is obtained using larger element sizes. If we compare quadratic elements with two times smaller linear elements, the computational time for quadratic elements is around two times shorter while the number of computational nodes is similar.

To summarize, both element types are suitable for solving bio-heat melanoma problems and can give accurate results using appropriate domain discretization. However, if the combination of accuracy and reduced computational time is the prevailing factor, quadratic elements are preferred. 

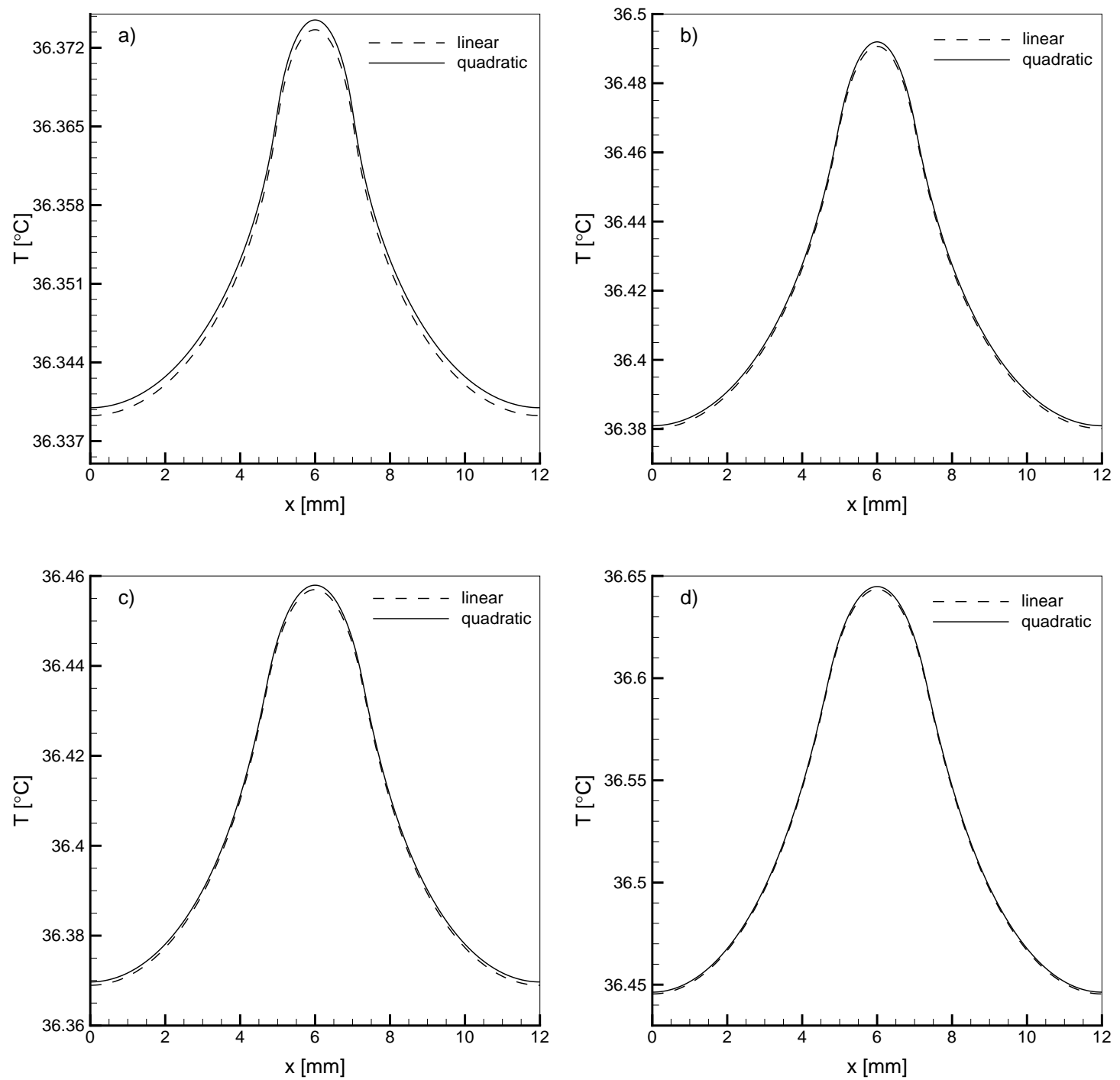

Figure 6: Skin surface temperature comparing linear and quadratic elements for the same element size of $0.05 m m$; a) Clark II $-\omega_{b, m 1}$, b) Clark II $-\omega_{b, m 2}$, c) Clark IV $-\omega_{b, m 1}$ and d) Clark IV $-\omega_{b, m 2}$.

\subsection{Transient results}

From the steady-state analysis, we concluded that quadratic elements can achieve greater numerical accuracy and are therefore chosen for the transient simulations. In this section, we will be testing the time domain discretization and the difference between elliptic and parabolic formulations.

Figure 7 shows the transient skin surface temperature response for the Clark II and IV melanoma at higher blood perfusion rates and surrounding tissue, using the elliptic formulation, element size of $d x=0.5 \mathrm{~mm}$ and different time steps; $d t=5 \mathrm{~s}$ and $d t=1 \mathrm{~s}$. As can be seen, the difference between the temperature in the melanoma region and surrounding skin is noticeable, especially in the case of higher blood perfusion. This is one of the reasons why Çetingül and Herman [3] proposed the cooling-rewarming test for melanoma detection. However, from the numerical point of view, the difference between calculated responses using different time steps is minimal, and in this form (curves are 


\begin{tabular}{c|cc|cc|cc|cc}
$d x$ & \multicolumn{5}{|c|}{ Clark II } & \multicolumn{4}{c}{ Clark IV } \\
{$[\mathrm{mm}]$} & \multicolumn{4}{|c|}{$R M S\left(10^{-5}\right)$} & \multicolumn{4}{c}{$R M S\left(10^{-5)}\right.$} \\
& lin. & quad. & lin. & quad. & lin. & quad. & lin. & quad. \\
& $\omega_{b, m 1}$ & $\omega_{b, m 1}$ & $\omega_{b, m 2}$ & $\omega_{b, m 2}$ & $\omega_{b, m 1}$ & $\omega_{b, m 1}$ & $\omega_{b, m 2}$ & $\omega_{b, m 2}$ \\
\hline 1.0 & 1.6857 & 0.2231 & 3.3106 & 0.6449 & 2.7274 & 1.0112 & 11.1390 & 1.6191 \\
0.5 & 1.7290 & 0.1159 & 1.6782 & 0.2510 & 1.4820 & 0.2689 & 4.3256 & 0.3497 \\
0.2 & 2.2272 & 0.0526 & 2.7623 & 0.0761 & 2.0607 & 0.1201 & 1.9873 & 0.0834 \\
0.1 & 2.1336 & 0.0389 & 2.7888 & 0.0331 & 2.5613 & 0.0484 & 3.2497 & 0.0283 \\
0.05 & 2.0925 & 0.0391 & 2.7487 & 0.0265 & 2.3246 & 0.0314 & 2.9424 & 0.0052 \\
0.03 & 1.9572 & - & 2.5144 & - & 1.9949 & - & 2.5005 & -
\end{tabular}

Table 3: RMS error of skin surface temperature compared to the solution obtained by quadratic element size $d x=0.03 \mathrm{~mm}$.

\begin{tabular}{c|c|c|c|c}
$d x$ & \multicolumn{2}{|c|}{ Clark II } & \multicolumn{2}{c}{ Clark IV } \\
{$[\mathrm{mm}]$} & linear & quadratic & linear & quadratic \\
\hline 1.0 & 0.33 & 0.91 & 0.31 & 0.84 \\
0.5 & 0.77 & 2.27 & 0.75 & 2.18 \\
0.2 & 5.10 & 19.01 & 5.00 & 20.01 \\
0.1 & 40.64 & 229.1 & 45.87 & 210.3 \\
0.05 & 587.7 & 3088.2 & 614.2 & 3077.6 \\
0.03 & 4344.5 & 22110 & 4527.1 & 22422
\end{tabular}

Table 4: CPU time in seconds [s] for steady-state simulation.

practically identical) is not appropriate for evaluating the numerical accuracy of the individual formulations or time steps. Therefore, the effect of different time steps will be presented in the form of skin temperature distribution at different times; $t=70 \mathrm{~s}$ and $t=150 \mathrm{~s}$, as can be seen in Figures 8 and 9. In this form, the temperature difference using different time steps can be easily observed and a time step analysis can be made. As can be observed, when reducing the time step, the numerical solution converges towards the near analytical one. This is especially visible at time $t=150 \mathrm{~s}$, where the time derivative of temperature is smaller compared to the time $t=70 \mathrm{~s}$, because the solution is converging toward the steady-state condition.

We also investigated the effect of element size on time-dependent solutions, keeping the time step constant. The graphical representation of results is omitted here, because the solution difference is minimal and not visible. Therefore, we can conclude that the timedependent solution is independent of the domain discretization. However, the analysis of the $R M S$ error compared to the smaller element size, shown in Table 5 for time steps $d t=1 s$ and $d t=0.1 s$, shows this difference. For the transient results the $R M S$ error has been calculated using the following equation

$$
R M S=\sqrt{\frac{1}{n_{j} \cdot n_{t}} \cdot \sum_{j=1}^{n_{j}} \sum_{k=1}^{n_{t}} \frac{\left(T_{j, k}-T_{a, j, k}\right)^{2}}{T_{a, j, k}^{2}}},
$$

where $n_{t}$ represents the number of time steps or the number of evaluated temperatures in 

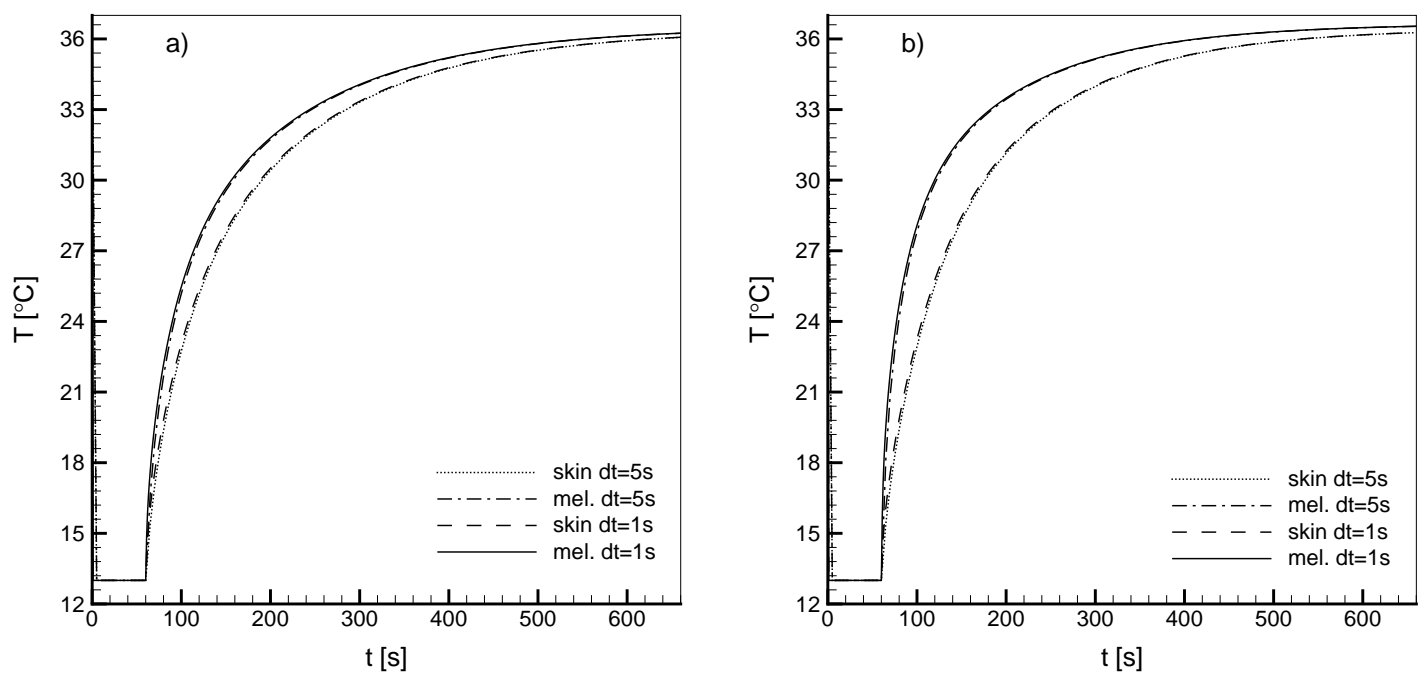

Figure 7: Skin surface temperature response at the center of melanoma (mel.) and for the surrounding tissue (skin); a) Clark II and b) Clark IV melanoma.

time. As can be seen from Table 5, the RMS error is reduced when using smaller element sizes for both time steps and is in the range of $10^{-5}$, which means numerical accurate results even for the element size of $d x=0.5 \mathrm{~mm}$.

\begin{tabular}{c|c|c|c|c|c}
$d t$ & $d x$ & \multicolumn{2}{|c|}{ Clark II } & \multicolumn{2}{c}{ Clark IV } \\
{$[s]$} & {$[m m]$} & \multicolumn{2}{|c}{$R M S\left(10^{-5}\right)$} & \multicolumn{2}{c}{$\operatorname{RMS}\left(10^{-5}\right)$} \\
& & $\omega_{b, m 1}$ & $\omega_{b, m 2}$ & $\omega_{b, m 1}$ & $\omega_{b, m 2}$ \\
\hline 1.0 & 0.5 & 1.8589 & 5.9859 & 3.6398 & 10.5990 \\
& 0.2 & 0.3189 & 0.8346 & 0.6542 & 1.4264 \\
\hline 0.1 & 0.5 & 2.1012 & 6.7056 & 3.7519 & 11.0470 \\
& 0.2 & 0.3680 & 0.9367 & 0.6494 & 1.5065
\end{tabular}

Table 5: RMS error of skin surface temperature compared to the solution obtained by element size $d x=0.1 \mathrm{~mm}$.

Figures 10 and 11 show the time step analysis using the parabolic formulation for all four cases, at times $t=70 \mathrm{~s}$ and $t=150 \mathrm{~s}$. We can quickly observe the numerical advantage and accuracy of the parabolic formulation, as the solution is less sensitive to the time step compared to the elliptic formulation. Comparing the elliptic and parabolic formulation results, we observe that the same numerical accuracy can be obtained using a larger time step for the parabolic formulation, which means much shorter computational times for direct transient bio-heat problems.

A detailed analysis comparing different formulations is shown in Figures 12 and 13, together with the numerically best solution. The results have been obtained for element size of $d x=0.5 \mathrm{~mm}$ and time step $d t=1 \mathrm{~s}$, while the numerically best solution has been obtained using the parabolic formulation and the finest domain and time discretization used in this study; $d x=0.1 \mathrm{~mm}$ and $d t=0.1 \mathrm{~s}$. As seen from the comparison of Figures 10 and 8, we can also observe the numerical advantage of using the parabolic formulation, where for the time step of $d t=1 s$ we nearly obtained the numerically best solution, 

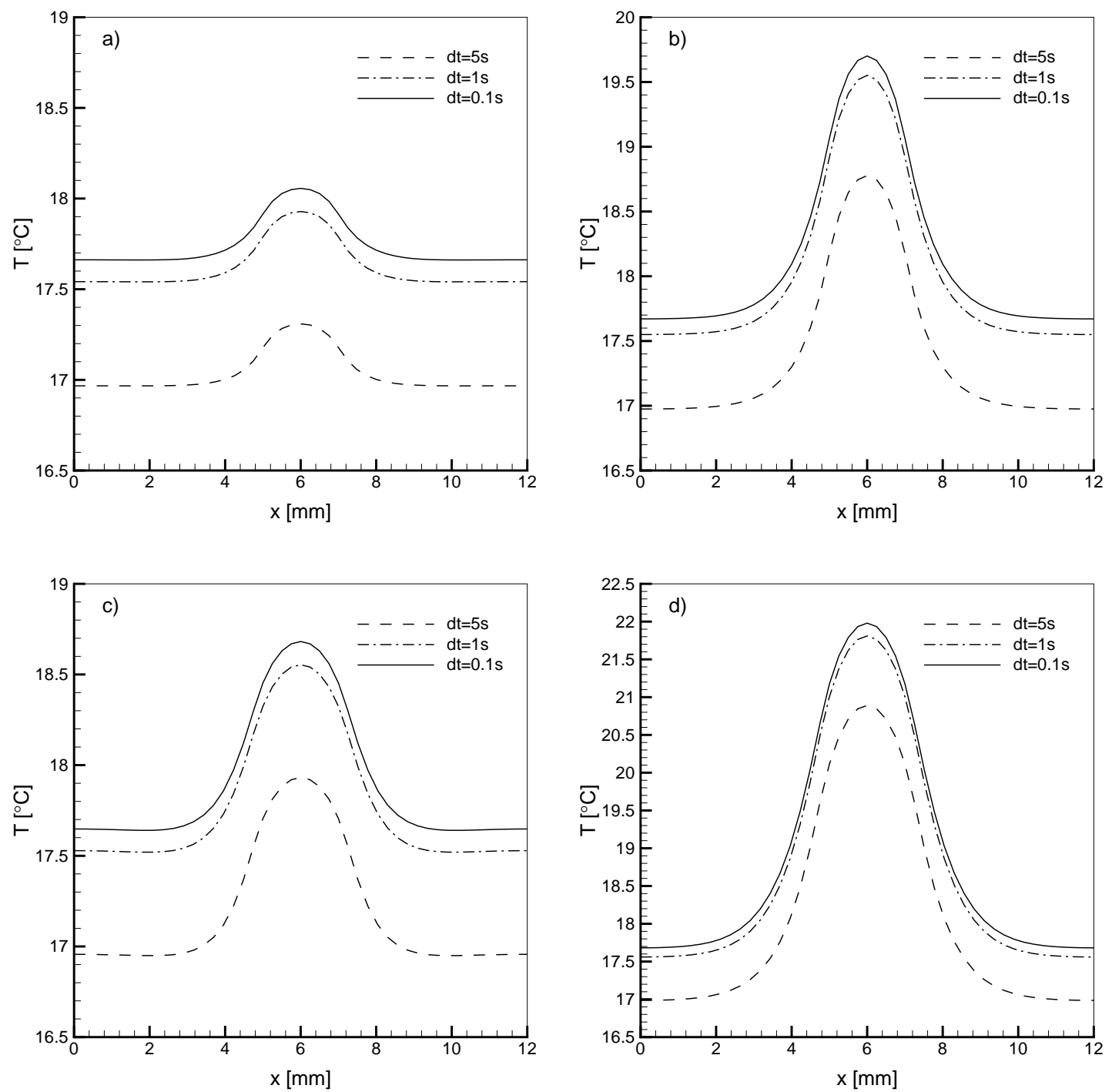

Figure 8: Skin surface temperature at time $t=70 \mathrm{~s}$ using elliptic formulation and different time steps; a) Clark II $-\omega_{b, m 1}$, b) Clark II $-\omega_{b, m 2}$, c) Clark IV $-\omega_{b, m 1}$ and d) Clark IV $-\omega_{b, m 2}$.

especially for later times $t>100 s$.

Under the cooling-rewarming test, the temperature difference at the skin surface above the tumor and healthy tissue is now much larger than from the steady-state conditions, producing larger temperature gradients. Therefore, the domain size or prescribed boundary condition can affect the numerical accuracy of the simulation. A domain size analysis is omitted from this paper as the focus is on assessing the performance of subdomain BEM elliptic and parabolic approaches.

The difference between numerical solutions for Clark IV melanoma and higher blood perfusion rate (Figure $13 \mathrm{~d}$ ) can be deceiving, because of the different temperature scale. Therefore, a detailed analysis of the numerical accuracy of different time steps, element sizes and formulations is presented through the calculated $R M S$ error in Table 6 for the elliptic formulation and Table 7 for the parabolic formulation, and the related computational time in Table 8. The $R M S$ error has been calculated based on the best numerical 

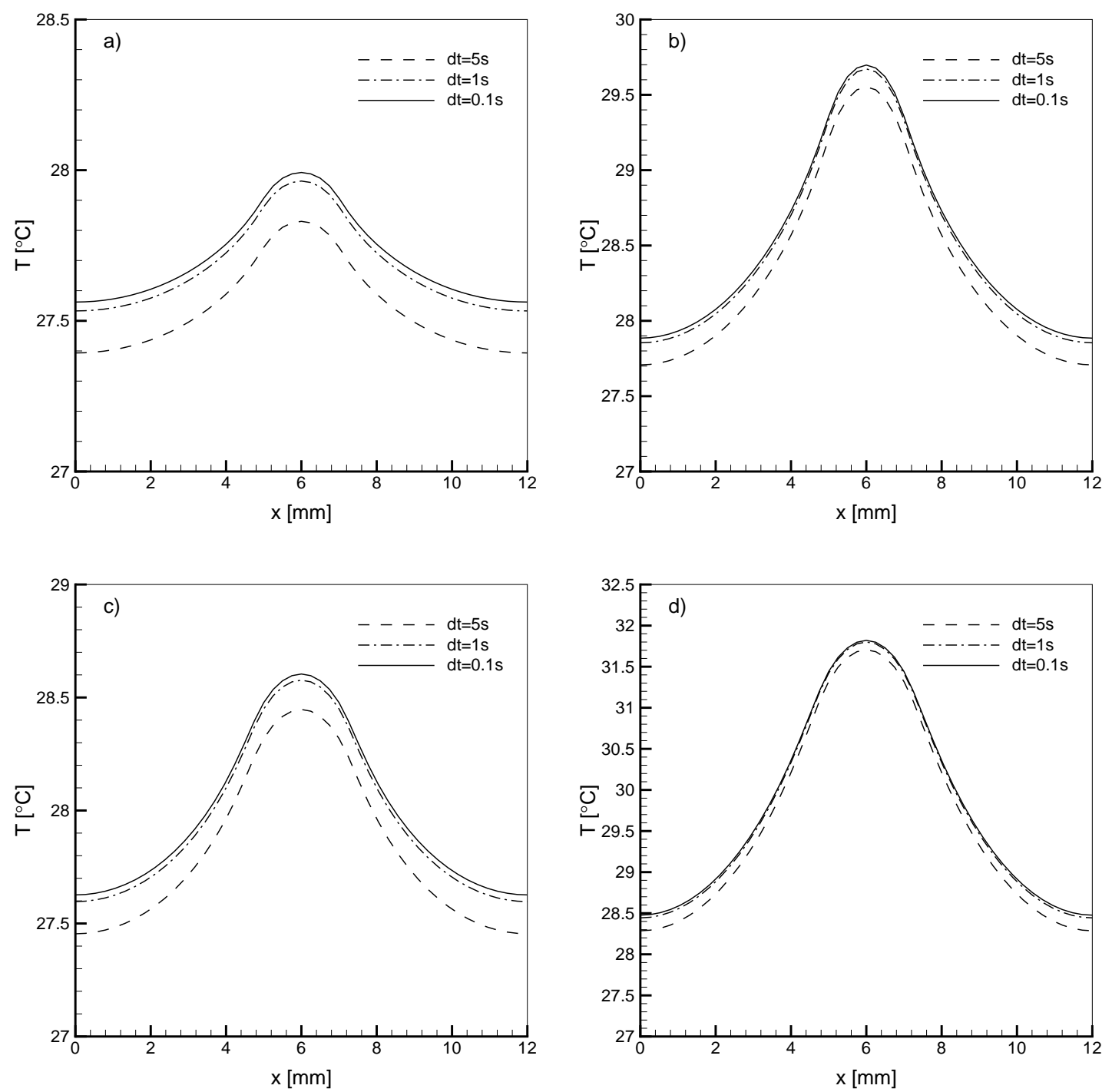

Figure 9: Skin surface temperature at time $t=150 \mathrm{~s}$ using elliptic formulation and different time steps; a) Clark II $-\omega_{b, m 1}$, b) Clark II $-\omega_{b, m 2}$, c) Clark IV $-\omega_{b, m 1}$ and d) Clark IV $-\omega_{b, m 2}$.

results obtained by the parabolic formulation with the finest domain and time discretization. The $R M S$ error lies in the region of $10^{-3}-10^{-4}$ for the time discretization, therefore the $R M S$ error shown in Table 5 for certain time steps indicates mesh independent results, as can be seen from Tables 6 and 7 . The $R M S$ error does not change by reducing the element size, however it reduces by reducing the time step. Table 7 shows that the $R M S$ error slightly increases in some cases when the element size is reduced. The reason for this increase can be traced to the comparison between the numerical solution and the best obtained numerical solution. Although that numerical solution is mesh independent for the same time step, some slight deviation is obtained. If we could compare the numerical solutions to the exact one, the $R M S$ error would show uniform convergence when reducing the element size for a given time step. By comparing the $R M S$ error achieved by the elliptic and parabolic formulations, we observe a lower error for the parabolic formulation or, for the same level of error, the time step can be ten times larger than 

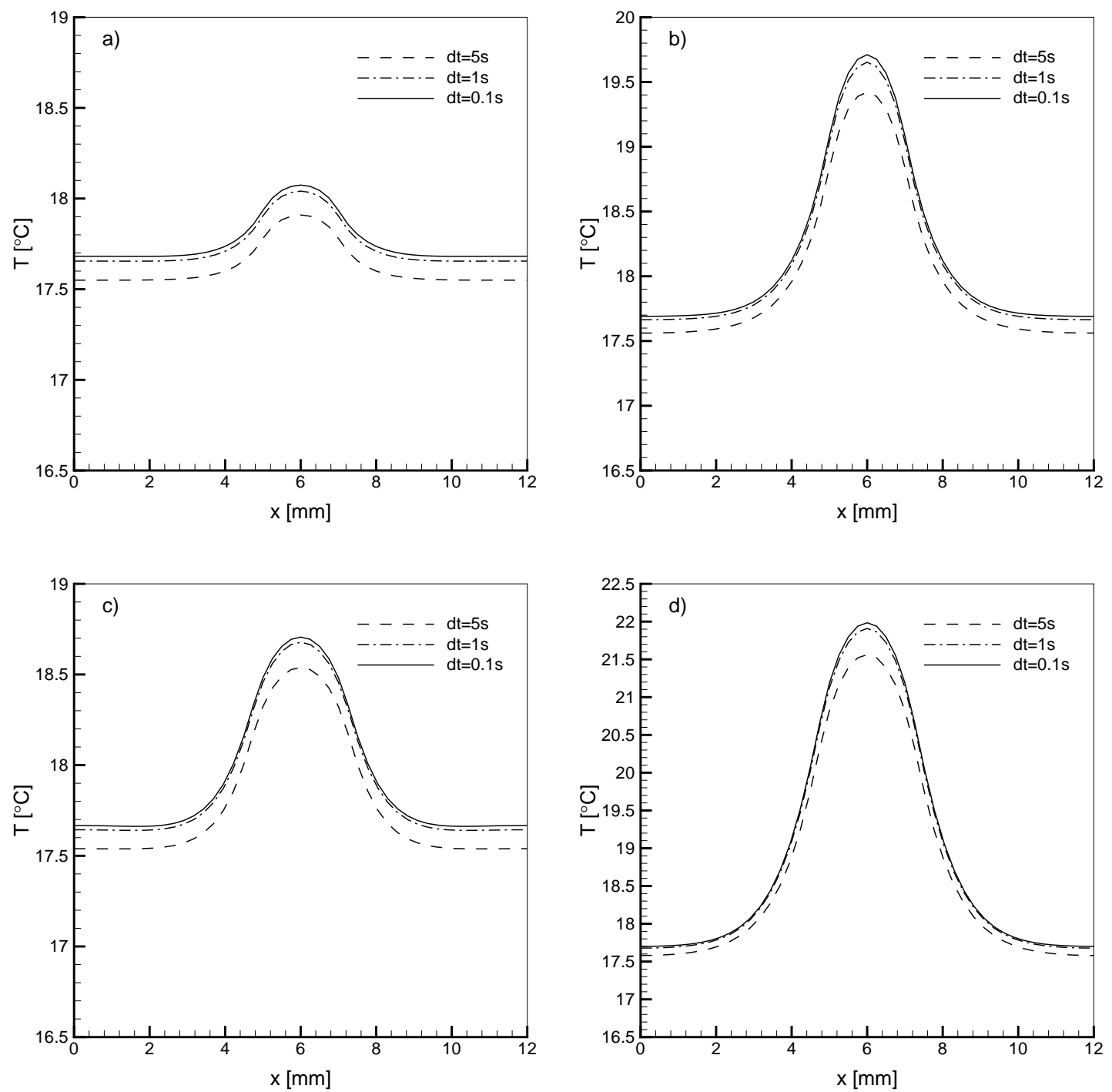

Figure 10: Skin surface temperature at time $t=70 \mathrm{~s}$ using parabolic formulation and different time steps; a) Clark II $-\omega_{b, m 1}$, b) Clark II $-\omega_{b, m 2}$, c) Clark IV $-\omega_{b, m 1}$ and d) Clark IV $-\omega_{b, m 2}$.

the time step for the elliptic formulation. Therefore, the speed-up for achieving the same level of error is between 11-22, looking at Table 8 for element size of $0.5 \mathrm{~mm}$. This is a large saving in computational time, which is especially important for solving inverse problems. Comparing the numerical accuracy and computational time, we suggest the use of element size $0.5 \mathrm{~mm}$ and time step of $1 \mathrm{~s}$, because even by lowering the time step, the $R M S$ error does not decrease drastically.

\section{Conclusion}

This work focuses on solving the direct bio-heat melanoma problem as a step to providing a useful diagnostic tool, taking into account the numerical accuracy of solutions and their computational speed. The numerical solvers derived in this work are to be used for solving different inverse bio-heat problems, such as non-invasive determination of the tumours to 

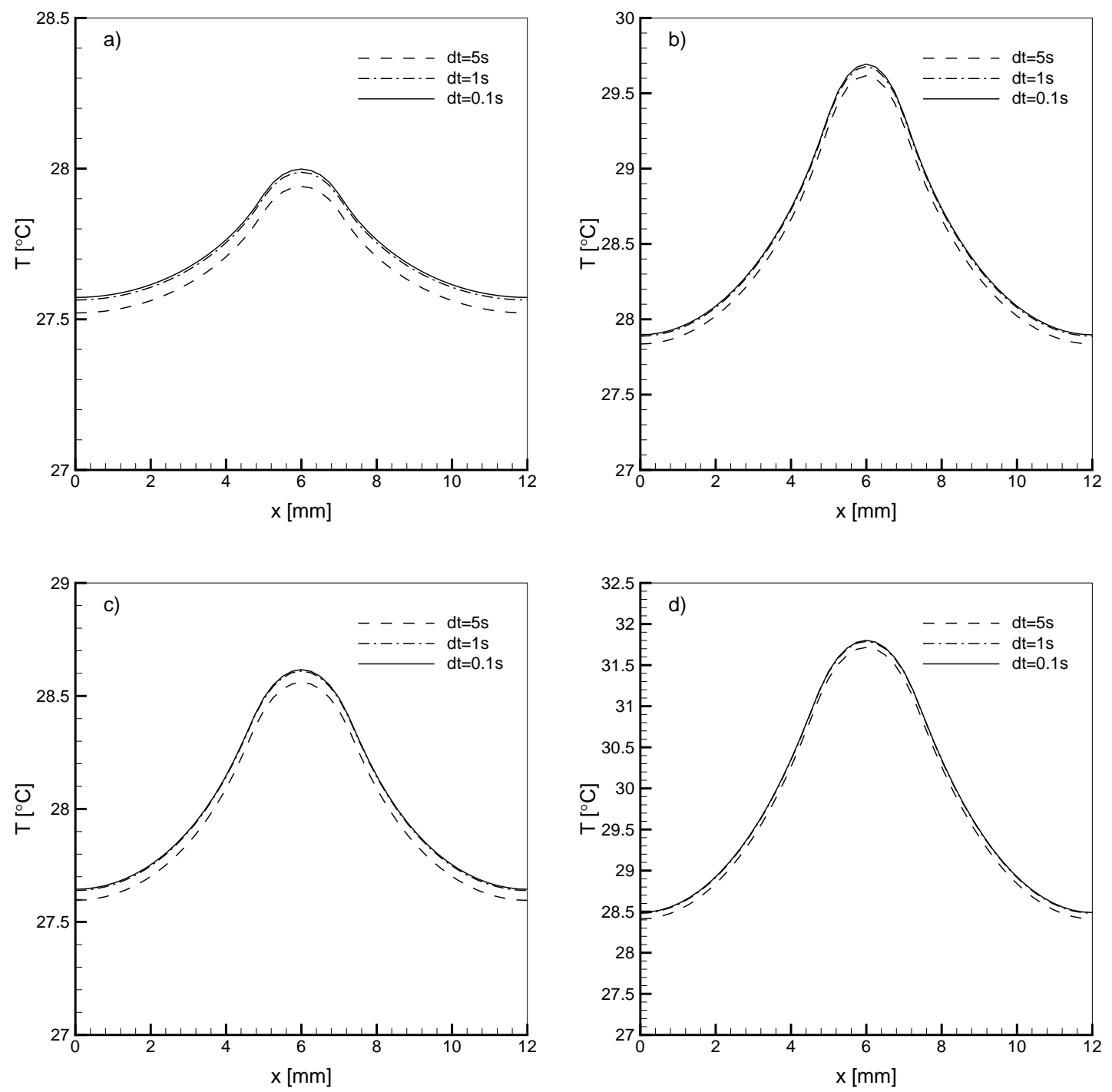

Figure 11: Skin surface temperature at time $t=150 \mathrm{~s}$ using parabolic formulation and different time steps; a) Clark II $-\omega_{b, m 1}$, b) Clark II $-\omega_{b, m 2}$, c) Clark IV $-\omega_{b, m 1}$ and d) Clark IV $-\omega_{b, m 2}$.

optimize hyperthermia or cryogenic treatments, for which an accurate and fast numerical solver is needed. The numerical accuracy of direct bio-heat solutions is especially needed in the case of deterministic (gradient based) optimization techniques, where the objective function has to be as smooth as possible. Unconditionally smooth objective functions can only be obtained in the case of known analytical solutions for the direct problem, while in most cases an analytical solution is not known and is therefore obtained numerically. In our case, the objective function is discrete resulting in incorrect gradients unless the numerical error is minimized, this being the main aim of this study.

The proposed numerical solver is based on the subdomain BEM approach, where different elements have been used as well as different BEM formulations of the governing equation. The elliptic formulation is based on the use of the Laplace fundamental solution and a FD scheme for the time derivative approximation, while the parabolic formulation uses the time-dependent fundamental solution of the diffusion equation with linear time 

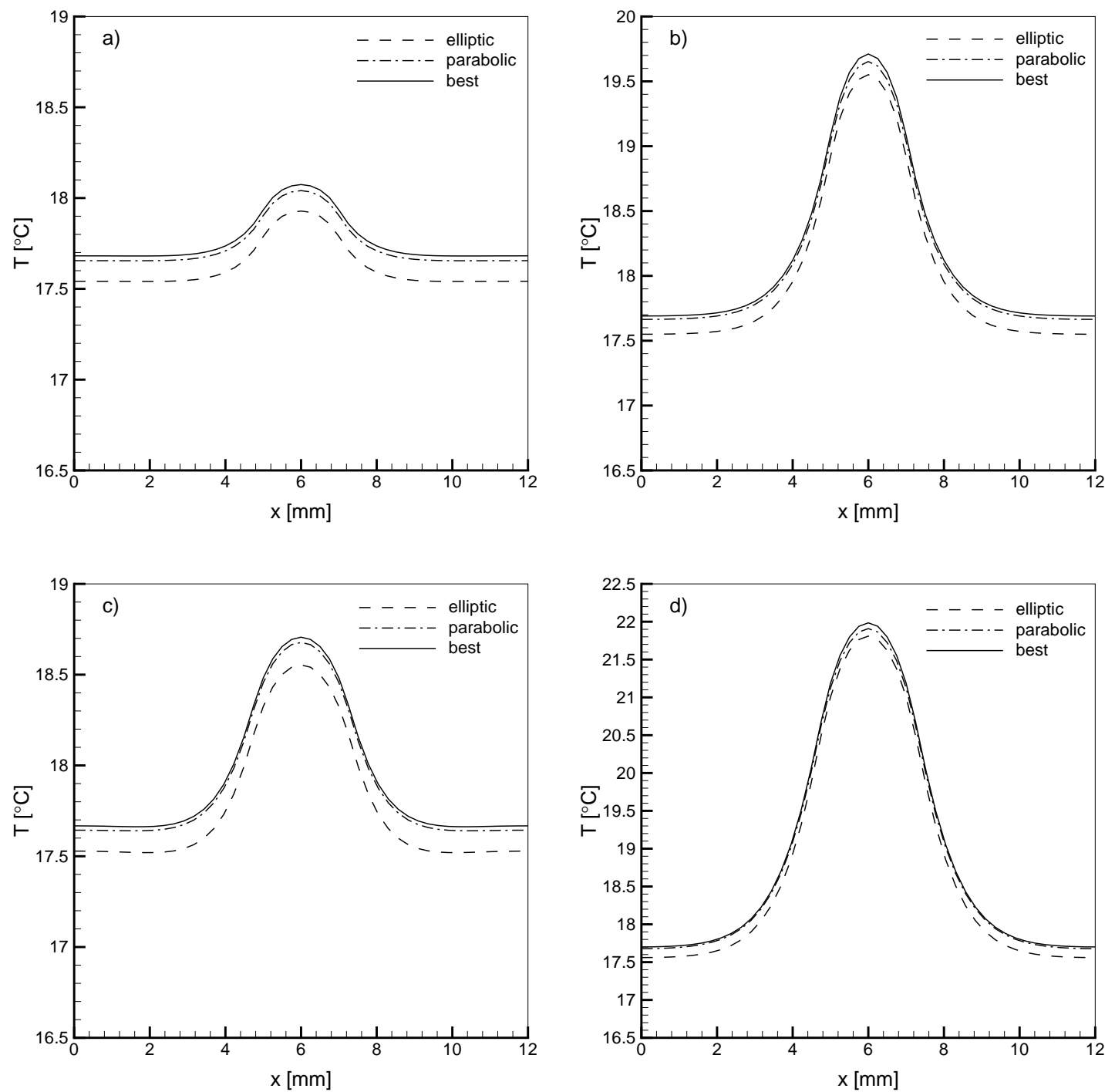

Figure 12: Skin surface temperature at time $t=70 \mathrm{~s}$ using element size of $d x=0.5 \mathrm{~mm}$, time step of $d t=1 \mathrm{~s}$ and different formulations together with numerically best solution; a) Clark II $-\omega_{b, m 1}$, b) Clark II $-\omega_{b, m 2}$, c) Clark IV $-\omega_{b, m 1}$ and d) Clark IV $-\omega_{b, m 2}$.

interpolation for the temperature and constant time interpolation for the normal derivative and heat source. Numerical accuracy is evaluated for two different elements; linear and quadratic. Linear elements use a linear interpolation function for the temperature and heat source, while quadratic elements use quadratic interpolations; however, both element types use constant interpolation for the normal derivative of the temperature field. For the parabolic formulation, we evaluated the time integrals analytically. Both formulations need a single evaluation of the domain and boundary integrals during the simulation, therefore they are equally computational demanding.

The proposed approaches using different BEM formulations and element types with mixed interpolation functions have been tested on steady-state as well as transient bioheat melanoma problems, considering different tissue layers. Four test cases have been analyzed, using different sizes of melanoma; Clark II and Clark IV, as well as different blood perfusion rates. The main aim was to evaluate the numerical accuracy of different 

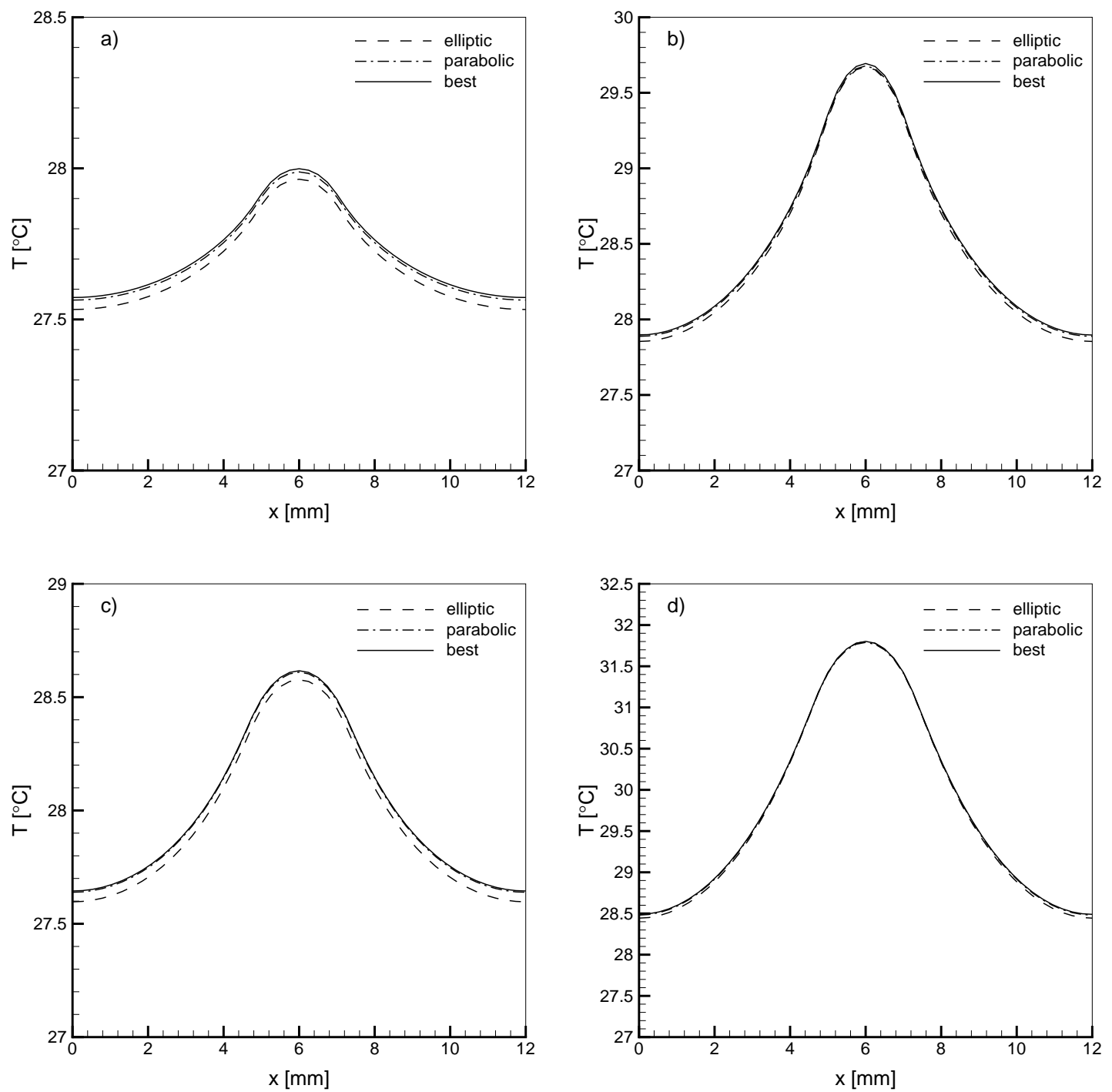

Figure 13: Skin surface temperature at time $t=150 \mathrm{~s}$ using element size of $d x=0.5 \mathrm{~mm}$, time step of $d t=1 \mathrm{~s}$ and different formulations together with numerically best solution; a) Clark II $-\omega_{b, m 1}$, b) Clark II $-\omega_{b, m 2}$, c) Clark IV $-\omega_{b, m 1}$ and d) Clark IV $-\omega_{b, m 2}$.

element types and proposed formulations, and to assess which numerical scheme is the most accurate and fast, and is thus appropriate for solving inverse problems.

The elliptic and parabolic formulations gave essentially the same results for steadystate problems, therefore only the element type and domain discretization have been analyzed. The results showed that quadratic elements achieved more accurate numerical results using larger elements than linear ones, for which the computational times are smaller. Therefore, quadratic elements have been used in the transient simulation analysis where we assessed the time and domain discretizations but, most importantly, the elliptic and parabolic formulations. The results show superior performance of the parabolic formulation, as the target numerical accuracy can be achieved using much larger time steps which translates into faster computational times. The results also showed that quadratic elements gave nearly mesh-independent transient results for which larger element sizes could be used. 


\begin{tabular}{|c|c|c|c|c|c|c|c|c|c|}
\hline & \multirow[b]{2}{*}{$d x[m m]$} & \multicolumn{4}{|c|}{$\begin{array}{c}\omega_{b, m 1} \\
d t[s]\end{array}$} & \multicolumn{4}{|c|}{$\begin{array}{c}\omega_{b, m 2} \\
d t[s]\end{array}$} \\
\hline & & 5.0 & 1.0 & 0.5 & 0.1 & 5.0 & 1.0 & 0.5 & 0.1 \\
\hline \multirow{4}{*}{ Clark II } & 1.0 & 8.1783 & 2.0019 & 1.0648 & 0.2165 & 8.5443 & 2.1599 & 1.1945 & 0.4126 \\
\hline & 0.5 & 8.1939 & 2.0101 & 1.0698 & 0.1997 & 8.5396 & 2.1293 & 1.1415 & 0.2280 \\
\hline & 0.2 & 8.1997 & 2.0138 & 1.0729 & 0.2025 & 8.5426 & 2.1276 & 1.1379 & 0.2144 \\
\hline & 0.1 & 8.2014 & 2.0152 & 1.0741 & 0.2019 & 8.5443 & 2.1283 & 1.1383 & 0.2121 \\
\hline \multirow{4}{*}{ Clark IV } & 1.0 & 8.2458 & 2.0748 & 1.1326 & 0.3028 & 9.0951 & 2.4718 & 1.4582 & 0.6657 \\
\hline & 0.5 & 8.2737 & 2.0716 & 1.1160 & 0.2226 & 9.0558 & 2.3627 & 1.2922 & 0.2849 \\
\hline & 0.2 & 8.2910 & 2.0773 & 1.1197 & 0.2241 & 9.0518 & 2.3487 & 1.2758 & 0.2479 \\
\hline & 0.1 & 8.2945 & 2.0788 & 1.1207 & 0.2509 & 9.0575 & 2.3507 & 1.2763 & 0.2547 \\
\hline
\end{tabular}

Table 6: $R M S$ error $\left(\cdot 10^{-3}\right)$ of skin surface temperature response using elliptic formulation compared to the numerically best available solution.

\begin{tabular}{cc|cccc|ccccc} 
& & \multicolumn{5}{|c|}{$\omega_{b, m 1}$} & \multicolumn{5}{c}{$\omega_{b, m 2}$} \\
$d t[s]$ & \multicolumn{4}{c}{$d t[s]$} \\
& & $d x[m m]$ & 5.0 & 1.0 & 0.5 & 0.1 & 5.0 & 1.0 & 0.5 & 0.1 \\
\hline \multirow{3}{*}{ Clark II } & 1.0 & 1.2297 & 0.3865 & 0.4247 & 0.1263 & 1.6723 & 0.4868 & 0.4990 & 0.1146 \\
& 0.5 & 1.7620 & 0.3950 & 0.1876 & 0.1642 & 2.1615 & 0.4802 & 0.2312 & 0.1429 \\
& 0.2 & 2.2372 & 0.5257 & 0.2450 & 0.0146 & 2.6183 & 0.6070 & 0.2849 & 0.0159 \\
& 0.1 & 2.3961 & 0.6209 & 0.3159 & - & 2.7736 & 0.7005 & 0.3529 & - \\
\hline \multirow{3}{*}{ Clark IV } & 1.0 & 1.1352 & 0.5745 & 0.5367 & 0.6882 & 2.3555 & 0.7499 & 0.6217 & 0.5601 \\
& 0.5 & 1.6426 & 0.4015 & 0.2723 & 0.3191 & 2.7486 & 0.6165 & 0.3113 & 0.1861 \\
& 0.2 & 2.2742 & 0.5273 & 0.2438 & 0.0378 & 3.2127 & 0.7562 & 0.3596 & 0.0307 \\
& 0.1 & 2.4572 & 0.6413 & 0.3256 & - & 3.3718 & 0.8534 & 0.4275 & -
\end{tabular}

Table 7: RMS error $\left(\cdot 10^{-3}\right)$ of skin surface temperature response using parabolic formulation compared to the numerically best available solution.

\begin{tabular}{cc|cccc|ccccc} 
& & \multicolumn{5}{|c|}{ Clark II } & \multicolumn{5}{c}{ Clark IV } \\
& & \multicolumn{4}{|c|}{$d t[s]$} & \multicolumn{5}{c}{$d t[s]$} \\
\multirow{3}{*}{ Elliptic } & $d x[\mathrm{~mm}]$ & 5.0 & 1.0 & 0.5 & 0.1 & 5.0 & 1.0 & 0.5 & 0.1 \\
\hline \multirow{3}{*}{ Parabolic } & 1.0 & 0.0041 & 0.0243 & 0.0533 & 0.2842 & 0.0035 & 0.0224 & 0.0503 & 0.2160 \\
& 0.5 & 0.0130 & 0.0745 & 0.1593 & 0.7807 & 0.0129 & 0.0716 & 0.1484 & 0.7388 \\
& 0.2 & 0.1570 & 0.7786 & 1.5043 & 8.0013 & 0.1517 & 0.7472 & 1.5216 & 7.7555 \\
& 0.1 & 1.9833 & 9.6309 & 19.545 & 95.67 & 1.8649 & 9.3336 & 18.779 & 93.94 \\
\hline & 0.5 & 0.0019 & 0.0062 & 0.0108 & 0.0481 & 0.0015 & 0.0047 & 0.0087 & 0.0367 \\
& 0.2 & 0.1678 & 0.0345 & 0.0643 & 0.2928 & 0.0078 & 0.0304 & 0.0569 & 0.2580 \\
& 0.1 & 2.4071 & 10.934 & 21.188 & 109.98 & 2.2847 & 10.308 & 20.120 & 105.05
\end{tabular}

Table 8: CPU time in $[h]$ using elliptic or parabolic formulations. 
Based on the results of this work, we propose the use of the BEM parabolic formulation with quadratic elements for solving direct bio-heat melanoma problems. The corresponding results showed that coarser discretizations can be used for the same level of accuracy to the linear element type and elliptic formulation.

In conclusion, this work provides a detailed analysis of various bio-heat melanoma problems that can be used for benchmarking numerical formulation and serve as a basis for our future work in the field of bio-heat transfer simulations, from direct problems to inverse ones such as non-invasive tumour detection and parameter evaluation.

\section{References}

[1] A. Bhowmik, R. Repaka, Estimation of growth features and thermophysical properties of melanoma within 3-D human skin using genetic algorithm and simulated annealing, International Journal of Heat and Mass Transfer 98 (2016) 81-95.

[2] J. M. Luna, A. Hernández Guerrero, R. Romero Méndez, J. L. Luviano Ortiz, Solution of the inverse bio-heat transfer problem for a simplified dermatological application: Case of skin cancer, Ingeniería Mecánica, Tecnología y Desarrollo 4 (6) (2014) $219-228$.

[3] M. P. Çetingül, C. Herman, Quantification of the thermal signature of a melanoma lesion, International Journal of Thermal Sciences 50 (4) (2011) 421-431.

[4] P. W. Partridge, L. C. Wrobel, A coupled dual reciprocity BEM/genetic algorithm for identification of blood perfusion parameters, International Journal of Numerical Methods for Heat \& Fluid Flow 19 (1) (2009) 25-38.

[5] M. Paruch, E. Majchrzak, Identification of tumor region parameters using evolutionary algorithm and multiple reciprocity boundary element method, Engineering Applications of Artificial Intelligence 20 (5) (2007) 647-655.

[6] P. W. Partridge, L. C. Wrobel, An inverse geometry problem for the localisation of skin tumours by thermal analysis, Engineering Analysis with Boundary Elements 31 (10) (2007) 803-811.

[7] K. Das, S. C. Mishra, Simultaneous estimation of size, radial and angular locations of a malignant tumor in a 3-D human breast - A numerical study, Journal of thermal biology 52 (2015) 147-156.

[8] N. A. Kudryashov, K. E. Shilnikov, Numerical modeling and optimization of the cryosurgery operations, Journal of Computational and Applied Mathematics 290 (2015) 259-267.

[9] M. Ge, K. Chua, C. Shu, W. Yang, Analytical and numerical study of tissue cryofreezing via the immersed boundary method, International Journal of Heat and Mass Transfer 83 (2015) 1-10.

[10] K. K. Ramajayam, A. Kumar, A novel approach to improve the efficacy of tumour ablation during cryosurgery, Cryobiology 67 (2) (2013) 201-213. 
[11] C. Thaokar, Y. Rabin, Temperature field reconstruction for minimally invasive cryosurgery with application to wireless implantable temperature sensors and/or medical imaging, Cryobiology 65 (3) (2012) 270-277.

[12] D. Kumar, K. Rai, A study on thermal damage during hyperthermia treatment based on DPL model for multilayer tissues using finite element Legendre wavelet Galerkin approach, Journal of Thermal Biology 62 (2016) 170-180.

[13] S. Hassanpour, A. Saboonchi, Interstitial hyperthermia treatment of countercurrent vascular tissue: A comparison of Pennes, WJ and porous media bioheat models, Journal of Thermal Biology 46 (2014) 47-55.

[14] M. M. Attar, M. Haghpanahi, S. Amanpour, M. Mohaqeq, Analysis of bioheat transfer equation for hyperthermia cancer treatment, Journal of Mechanical Science and Technology 28 (2) (2014) 763-771.

[15] M. M. Paulides, P. R. Stauffer, E. Neufeld, P. F. Maccarini, A. Kyriakou, R. A. Canters, C. J. Diederich, J. F. Bakker, G. C. Van Rhoon, Simulation techniques in hyperthermia treatment planning, International Journal of Hyperthermia 29 (4) (2013) 346-357.

[16] A. Joukar, E. Nammakie, H. Niroomand-Oscuii, A comparative study of thermal effects of 3 types of laser in eye: 3D simulation with bioheat equation, Journal of Thermal Biology 49 (2015) 74-81.

[17] M. Jaunich, S. Raje, K. Kim, K. Mitra, Z. Guo, Bio-heat transfer analysis during short pulse laser irradiation of tissues, International Journal of Heat and Mass Transfer 51 (23) (2008) 5511-5521.

[18] M. F. Marqa, S. Mordon, N. Betrouni, Laser interstitial thermotherapy of small breast fibroadenomas: Numerical simulations, Lasers in Surgery and Medicine 44 (10) (2012) 832-839.

[19] J.-H. Kim, W. J. Williams, A. Coca, M. Yokota, Application of thermoregulatory modeling to predict core and skin temperatures in firefighters, International Journal of Industrial Ergonomics 43 (1) (2013) 115-120.

[20] J. E. Laszczyk, A. J. Nowak, Computational modelling of neonate's brain cooling, International Journal of Numerical Methods for Heat \& Fluid Flow 26 (2) (2016) $571-590$.

[21] C. B. Pereira, K. Heimann, M. Czaplik, V. Blazek, B. Venema, S. Leonhardt, Thermoregulation in premature infants: A mathematical model, Journal of Thermal Biology 62 (2016) 159-169.

[22] A. B. C. Silva, J. Laszczyk, L. C. Wrobel, F. L. Ribeiro, A. J. Nowak, A thermoregulation model for hypothermic treatment of neonates, Medical Engineering \& Physics 38 (9) (2016) 988-998.

[23] M. Ramšak, J. Ravnik, M. Zadravec, M. Hriberšek, J. Iljaž, Freeze-drying modeling of vial using BEM, Engineering Analysis with Boundary Elements 77 (2017) 145-156. 
[24] M. Ramšak, L. Škerget, A highly efficient multidomain BEM for multimillion subdomains, Engineering Analysis with Boundary Elements 43 (2014) 76 - 85.

[25] M. Ramšak, L. Škerget, Heat diffusion in fractal geometry cooling surface, Thermal Science 16 (4) (2012) 955-968.

[26] J. Ravnik, M. Hriberšek, J. Lupše, Lagrangian particle tracking in velocity-vorticity resolved viscous flows by subdomain BEM, Journal of Applied Fluid Mechanics 9 (3) (2016) 1533-1549.

[27] J. Ravnik, L. Škerget, Z. Žunič, Velocity-vorticity formulation for 3D natural convection in an inclined enclosure by BEM, International Journal of Heat and Mass Transfer 51 (17) (2008) 4517-4527.

[28] J. Iljaž, L. Škerget, Blood perfusion estimation in heterogeneous tissue using BEM based algorithm, Engineering Analysis with Boundary Elements 39 (2014) 75-87.

[29] L. Yan, C.-L. Fu, F.-L. Yang, The method of fundamental solutions for the inverse heat source problem, Engineering Analysis with Boundary Elements 32 (3) (2008) $216-222$.

[30] D. Young, C. Tsai, K. Murugesan, C. Fan, C. Chen, Time-dependent fundamental solutions for homogeneous diffusion problems, Engineering Analysis with Boundary Elements 28 (12) (2004) 1463-1473.

[31] B. T. Johansson, D. Lesnic, A method of fundamental solutions for transient heat conduction in layered materials, Engineering Analysis with Boundary Elements 33 (12) (2009) 1362-1367.

[32] T.-Y. Cheng, C. Herman, Analysis of skin cooling for quantitative dynamic infrared imaging of near-surface lesions, International Journal of Thermal Sciences 86 (2014) $175-188$.

[33] H. H. Pennes, Analysis of tissue and arterial blood temperatures in the resting human forearm, Journal of Applied Physiology 1 (2) (1948) 93-122.

[34] S. Weinbaum, L. Xu, L. Zhu, A. Ekpene, A new fundamental bioheat equation for muscle tissue: Part I - Blood perfusion term, Journal of biomechanical engineering 119 (3) (1997) 278-288.

[35] A. Bhowmik, R. Singh, R. Repaka, S. C. Mishra, Conventional and newly developed bioheat transport models in vascularized tissues: A review, Journal of Thermal Biology 38 (3) (2013) 107-125.

[36] F. Xu, K. Seffen, T. Lu, Non-Fourier analysis of skin biothermomechanics, International Journal of Heat and Mass Transfer 51 (9) (2008) 2237-2259.

[37] A. Nakayama, F. Kuwahara, A general bioheat transfer model based on the theory of porous media, International Journal of Heat and Mass Transfer 51 (11) (2008) 3190-3199. 
[38] L. C. Wrobel, The boundary element method, applications in thermo-fluids and acoustics, Vol. 1, John Wiley \& Sons, 2002.

[39] D. Barry, J.-Y. Parlange, L. Li, Approximation for the exponential integral (Theis well function), Journal of Hydrology 227 (1) (2000) 287-291. 